\title{
SOHO/SWAN OBSERVATIONS OF SHORT-PERIOD SPACECRAFT TARGET COMETS
}

\author{
M. R. Combi ${ }^{1}$, Y. LeE $^{1}$, T. S. PATeL ${ }^{1}$, J. T. T. MÄKInen ${ }^{2}$, J.-L. BertauX ${ }^{3}$, And E. Quémerais ${ }^{3}$ \\ ${ }^{1}$ Department of Atmospheric, Oceanic and Space Sciences, University of Michigan, 2455 Hayward Street, Ann Arbor, MI 48109-2143, USA; mcombi@ umich.edu \\ ${ }^{2}$ Finnish Meteorological Institute, P.O. Box 503, SF-00101 Helsinki, Finland \\ ${ }^{3}$ LATMOS, CNRS/INSU, Université de Versailles Saint-Quentin, 11 Bd d'Alembert, 78280 Guyancourt, France \\ Received 2010 April 29; accepted 2011 February 7; published 2011 March 8
}

\begin{abstract}
SWAN, the Solar Wind ANisotropies all-sky hydrogen Ly $\alpha$ camera on the Solar and Heliospheric Observatory spacecraft that makes all-sky images of interplanetary neutral hydrogen, has an ongoing campaign to make special observations of comets, both short- and long-period ones, in addition to the serendipitous observations of comets as part of the all-sky monitoring program. We report here on a study of several short-period comets that were detected by SWAN: 21P/Giacobini-Zinner (1998 and 2005 apparitions), 19P/Borrelly (2001 apparition), 81P/Wild 2 (1997 apparition), and 103P/Hartley 2 (1997 apparition). SWAN observes comets over long continuous stretches of their visible apparitions and therefore provides excellent temporal coverage of the water production. For some of the observations we are also able to analyze an entire sequence of images over many days to several weeks/months using our time-resolved model and extract daily average water production rates over continuous periods of several days to months. The short-term (outburst) and long-term behavior can be correlated with other observations. The overall long-term variation is examined in light of seasonal effects seen in the pre- to post-perihelion differences. For 21P/Giacobini-Zinner and 81P/Wild 2 the activity variations over each apparition were more continuously monitored but nonetheless consistent with previous observations. For 19P/Borrelly we found a very steep variation of water production rates, again consistent with some previous observations, and a variation over six months around perihelion that was reasonably consistent with the spin-axis model of Schleicher et al. and the illumination of the main active areas. During the 1997-1998 apparition of 103P/Hartley 2, the target comet of the EPOXI mission (the Deep Impact extended mission), we found a variation with heliocentric distance $\left(\sim r^{-3.6}\right)$ that was almost as steep as 19P/Borrelly and, given the small measured radius near aphelion, this places a number of possible constraints on the size, shape, and/or distribution active of areas on the surface.
\end{abstract}

Key words: comets: general - comets: individual (21P/Giacobini-Zinner, 19P/Borrelly, 81P/Wild 2, 103P/Hartley 2) - molecular processes

\section{INTRODUCTION}

Observations of hydrogen Ly $\alpha$ at $1215.7 \AA$ in comets and their interpretation are important. Atomic hydrogen is the most abundant species in the atmosphere (or coma) of a comet being produced in a photodissociation chain originating with water molecules and including intermediate $\mathrm{OH}$ radicals. Water is the most abundant volatile species in a comet's nucleus, and water sublimation controls the abundance and activity of the coma when comets are within $3 \mathrm{AU}$ from the Sun. Measurements of the abundance and distribution of hydrogen in the coma, when appropriately modeled, can provide a reliable measure of the water production rate and its variation in time in comets. Compositional information is normally compared to water, making it the most important for obtaining accurate production rates. Variations in production rate with time generally, and with heliocentric distance in particular can provide information about the volatile distribution and structure of the nucleus.

SWAN, the Solar Wind ANisotropies all-sky hydrogen Ly $\alpha$ camera, has been operating on the Solar and Heliospheric Observatory (SOHO) spacecraft since its launch in late 1995. The SWAN instrument was designed to observe the entire sky in H Ly $\alpha$ in order to obtain a global view of the variable interaction of the solar wind with the neutral interstellar hydrogen streaming through the solar system. From its viewpoint at the L1 Lagrange point between Earth and the Sun it obtains an unparallel view of the Sun, its large extended corona and the entire sky. For a more detailed description of the SWAN instrument on $\mathrm{SOHO}$, see Bertaux et al. (1995).
Because of the limited observing time available for synoptic cometary observations (or any solar system targets for that matter) with the Hubble Space Telescope (HST), compounded by the fact that many bright comets are often within the solar avoidance area of $H S T$ and by various problems with $H S T$ instruments, space-based observations, and in particular UV observations have been severely limited after 20 years when IUE was available. With its UV capability SWAN fills an important void in the monitoring of comets from space since early 1996.

Because of the large neutral hydrogen coma, SWAN can observe comets either in its full-sky mode, when comets are recorded during the regular full-sky interplanetary medium observations, or during special campaigns of comet-specific observations, when the region of the sky with a comet is specifically targeted and sometimes oversampled by the SWAN instrument field of view to yield a somewhat improved spatial resolution.

We report here on a study of SWAN observations of four short-period comets that are or have been spacecraft flyby targets: 21P/Giacobini-Zinner, 19P/Borrelly, 81P/Wild 2, and $103 \mathrm{P} /$ Hartley 2 . The single-image water production rates, those calculated using each single image, are presented for all. For two comets, we were able to use our time-resolved model (TRM) to analyze sequences of images simultaneously to obtain deconvolved daily average water production rates between the image snapshots. We discuss SWAN comet observations generally, followed by a discussion of the model analysis procedure, and then a discussion of each comet. In each of these sections we examine the variation of the water production rates 
with time and heliocentric distance, discuss their significance, and compare with other observations. The paper concludes with a general discussion of this campaign of analyses of SWAN observations of comets.

\section{OBSERVATIONS}

The observations presented in this paper result from two basic types of observational modes. SWAN routinely has observed the full sky three to seven times per week. Any comet bright enough will be registered on full-sky images. When a bright or otherwise interesting comet is expected, comet-specific observations have also been planned and made. The comet-specific images can improve the spatial resolution by oversampling and reduce noise by longer integration times over the full-sky images (Mäkinen \& Combi 2005). For the previous results on comet 1996 B2 (Hyakutake) the comet-specific images were more important when the comet was very close to Earth because of its large proper motion during the time the full-sky images are acquired. In that case, Combi et al. (2005) accounted for any motion issues in the daily average deconvolution with the TRM. These effects are included in the formal uncertainties in the single-image production rates, and as well in the daily averaged deconvolved results described in the following section. Tables giving the observational circumstances for all the SWAN images for each comet as well as the single-image production rates are presented below in the sections on each comet. The $g$-factor (reduced to a heliocentric distance of $1 \mathrm{AU}$ ) was derived from daily average observations by the SOLSTICE instruments on UARS and SORCE, TIMED SEE, which measures the solar UV irradiance. Missing and early data are supplemented with modeled values based on the measured F10.7 flux (Woods et al. 1996, 2000; McClintock et al. 2005). It is corrected to first order for the solar radiation difference between the face of the Sun seen by SOHO and that seen by the comet (Combi et al. 2000) and for the Doppler shift of the solar Ly $\alpha$ line profile caused by the heliocentric radial velocity of the comet.

A recent recalibration of the SWAN instrument has been performed comparing measurements of the Ly $\alpha$ brightness of the interplanetary medium with observations made with the $H S T$. The results presented here incorporate this new calibration. The SWAN calibration at Ly $\alpha$ is based on a comparison of SWAN with the Goddard High Resolution Spectrograph (GHRS) and Space Telescope Imaging Spectrograph (STIS) instruments on HST. The first inter-calibration measurements were performed in the summer of 1996a few months after the start of the SWAN observations. The GHRS instrument was used to measure the interplanetary line profile, and by integration, the interplanetary background brightness. However, the line of sight was in the downwind direction and the observation was performed close to solar minimum, which contributed to a rather small measured intensity of about $200 R$ (Clarke et al. 1998). From this measurement, an absolute calibration of SWAN at Ly $\alpha$ was derived (details in Quémerais \& Bertaux 2002). A second inter-calibration was performed in $2001 \mathrm{March}$, close to solar maximum, when STIS, which replaced GHRS on $H S T$, measured the interplanetary background brightness in the upwind direction. The measured intensity was close to $1400 R$ giving a much better signal-to-noise ratio (Quémerais et al. 2009). After some time, it appeared that the SWAN calibration factors deduced from both measurements were different. The ratio between the two values was close to 1.55 . Finally, a recent reexamination of the 1996 GHRS measurement showed that the good value is the second one and therefore the SWAN calibration factor was increased by a factor 1.55 for the 1996-1998 time period to correct for the initial value that was erroneously derived. All SWAN data sets have been corrected accordingly. The relative calibration of SWAN changes with time and has always been monitored with UV bright star observations. The effect of the change in calibration of SWAN on particular comet observations is discussed in Section 8 of this paper.

\section{TIME-RESOLVED MODEL}

We have used the TRM to extract the time history of the water production rate by analyzing the various series of images together for two of the comets, 19P/Borrelly and 81P/Wild 2. The details of the model are described in the paper by Mäkinen $\&$ Combi (2005). Because of its long lifetime $\left(\sim 1.5 \times 10^{6} \mathrm{~s}\right.$ at $1 \mathrm{AU}$ from the Sun), $\mathrm{H}$ atoms survive in the coma for $2-4$ weeks, during which time there are often available up to 10 or more SWAN images of the coma. The TRM uses a parameterized velocity distribution for $\mathrm{H}$ atoms in the coma, estimated from explicit calculations of the partial thermalization by the heavyatom coma (Combi \& Smyth 1988; Combi et al. 2000) and tracks their propagation out into the coma. The method then builds up a set of basis functions relating the production rate of water at the nucleus as a function of time to the distribution of photodissociated $\mathrm{H}$ atoms as seen on the sky plane images. In this paper, we present results of the water production rates in the standard single-image form for all of the comets as well as daily average water production rates extracted by the inversion scheme of the TRM for 19P/Borrelly and 81P/Wild 2.

\section{21P/GIACOBINI-ZINNER}

Comet 21P/Giacobini-Zinner (hereafter referred to as GZ) is a short-period Jupiter family comet with a perihelion distance of $1.038 \mathrm{AU}$. It has an orbital period of 6.5 years. GZ was the first comet to be sampled in situ in 1985 by the International Cometary Explorer (ICE) spacecraft, actually renamed the ISEE-3 satellite, which was diverted from measuring the solar wind interaction with Earth's magnetosphere to pass within $7800 \mathrm{~km}$ antisunward of the comet nucleus (von Rosenvinge et al. 1986). ICE was equipped with a variety of plasma field and charged particle instruments but no cameras or imaging spectrometers. According to the 85 comets survey classification of A'Hearn et al. (1995), comet GZ is the archetype member of the group depleted in $\mathrm{C}_{2}$ and $\mathrm{C}_{3}$ and somewhat depleted in $\mathrm{NH}$. This is consistent with the spectrophotometric survey of Fink \& Hicks (1996) and Fink (2009) who also found it depleted in $\mathrm{NH}_{2}$, agreeing with the earlier works of Konno \& Wyckoff (1989) and Beaver et al. (1990). The similar results for NH and $\mathrm{NH}_{2}$ would indicate that $\mathrm{GZ}$ is depleted in the likely parent $\mathrm{NH}_{3}$.

Infrared observations of parent molecules themselves are somewhat inconsistent or indicate that the production rate composition varies in time. Weaver et al. (1999) found an upper limit to the $\mathrm{CO} / \mathrm{H}_{2} \mathrm{O}$ ratio of only $0.2 \%-0.3 \%$, while Mumma et al. (2000) found a very measureable value of $10 \%$. For $\mathrm{C}_{2} \mathrm{H}_{6} /$ $\mathrm{H}_{2} \mathrm{O}$, Weaver et al. found a nominal to somewhat depleted level of $0.3 \%-0.5 \%$, while Mumma et al. found it clearly depleted at $0.09 \%-0.35 \%$. Mumma et al. interpreted these differences as indicating that these might be seasonal effects between the two different observation times and that the composition of the nucleus might be very heterogeneous.

In the two apparitions observed by SWAN, GZ reached perihelion on 1998 November 21.3205 and on 2005 July 2.7605. From the 1998 apparition useable SWAN observations were 


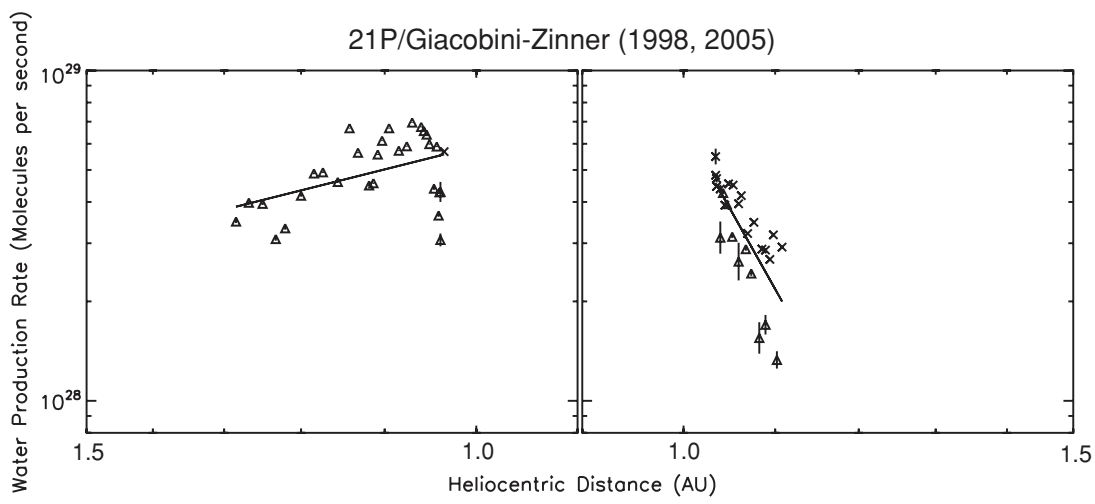

Figure 1. Single-image water production rates and fitted power-law distributions for comet 21P/Giacobini-Zinner. The triangles give the results from the 2005 apparition, perihelion on 2.76052005 July and the $x$ 's give the results from the 1998 apparition, perihelion on 21.32051998 November. The straight lines gives the best-fit power-law variation of the form $Q=5.9 \times 10^{28} r^{-1.7 \pm 0.4} \mathrm{~s}^{-1}$ for pre-perihelion and $Q=6.8 \times 10^{28} r^{-11.9 \pm 3.5} \mathrm{~s}^{-1}$ for post-perihelion.

obtained from November 20 through December 19 corresponding to a heliocentric distance range from 1.034 to 1.108 . From the 2005 apparition usable SWAN observations were obtained during the pre-perihelion period from April 16 to June 12 corresponding to a heliocentric distance range from $1.466 \mathrm{AU}$ before perihelion to $1.075 \mathrm{AU}$ after perihelion. The limitation after late June being that the comet was in the galactic plane with too much interference from background stars to distinguish the fairly faint comet. Figure 1 shows the single-image water production rates $(Q)$ of comet GZ plotted as a function of heliocentric distance $(r)$. A power-law fit of the form $Q=Q_{1 \mathrm{AU}} r^{p}$ gives a value at $Q=5.9 \times 10^{28} r^{-1.7 \pm 0.4} \mathrm{~s}^{-1}$ for pre-perihelion and $Q=$ $6.8 \times 10^{28} r^{-11.9 \pm 3.5} \mathrm{~s}^{-1}$ for post-perihelion. The latter is over a fairly small range of heliocentric distance. Comet GZ has a fairly well documented behavior of being more active at comparable heliocentric distances before perihelion than after. Because the production rate variation is likely dominated by such a peculiar seasonal effect, characterizing the mean power-law form for pre- and post-perihelion is rather meaningless. The single-image water production rates are listed, along with the observational circumstances in Table 1.

The water production rates are plotted as a function of time in days from perihelion in Figure 2. The overall variation, a function of time and the same sense of a pre- to post-perihelion asymmetry, agrees well with the early UV values that were summarized by Combi \& Feldman (1992). Included were their water production rates derived from $\mathrm{H} \mathrm{Ly} \alpha$ observations made with the IUE and from the Pioneer Venus Orbiter ultraviolet system (UVS) instrument (Combi et al. 1986) during the 1985 apparition, as well as observations of the OH (3090 ̊) emission from McFadden et al. (1987). All these observations were analyzed with reasonably consistent coma models and parameters.

The overall shape of the water production rate variation is also in quite good agreement with that derived by Schleicher et al. (1987) from ground-based photometric measurements of $\mathrm{OH}$ during the 1985 apparition in the UV at $310 \mathrm{~nm}$. The $\mathrm{OH}$ observations indicate that between 20 and 40 days after perihelion the rate of decrease in the water production rate flattens considerably after our last data point and remains at or just above $10^{28} \mathrm{~s}^{-1}$ even up to 90 days after perihelion. Therefore, the -11 slope in the post-perihelion production rate power law is just a short-lived feature likely from a seasonal effect on the nucleus for the 20 days after perihelion and not indicative of any long-term trend. One curious behavior is that typically, the water production rate variation, the water light

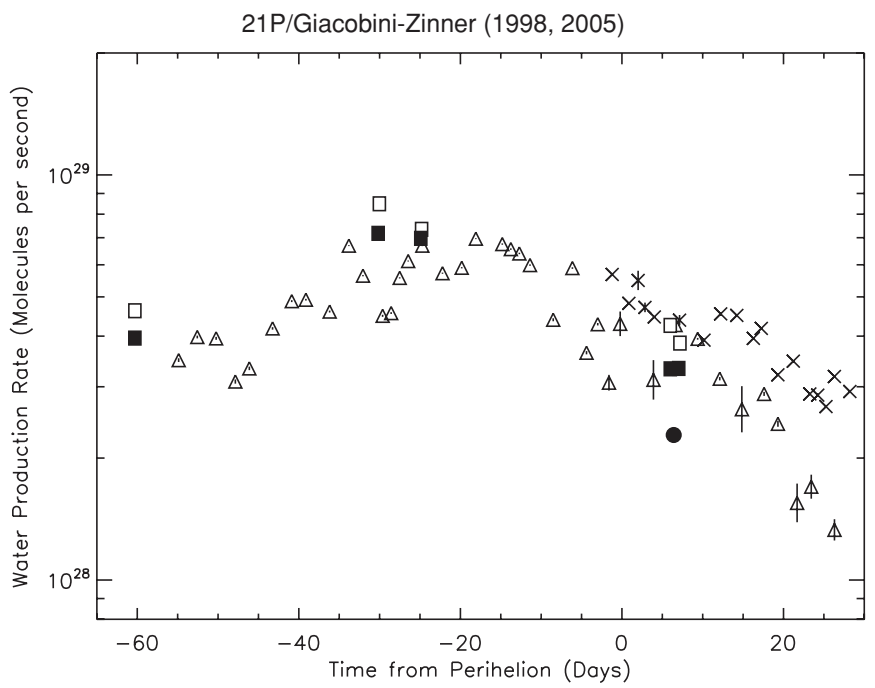

Figure 2. Water production rates of Comet 21P/Giacobini-Zinner as a function of time from perihelion in days. The triangles give the SWAN results from the 2005 apparition with perihelion on 2005 July 2, and the $x$ 's give the SWAN results from the 1998 apparition with perihelion on 1998 November 21. These are compared with various observations of the 1985 apparition relative to perihelion on 1985 September 11. The open squares are from IUE observations of $\mathrm{H} \mathrm{Ly} \alpha$ in (Combi \& Feldman 1992); the filled squares are from IUE observations of OH (3090 $⿱$ ) by McFadden et al. (1987); and the filled circle is from Pioneer Venus UVS observations of H Ly $\alpha$ by Combi et al. (1986).

curve if you like, resembles the visual light curve. For GZ, however, the peak visual magnitudes are seen very close to perihelion while the peak water production rate occurs from 20 to 25 days before perihelion, and the pre- to post-perihelion asymmetry in visual magnitude is not nearly as large as is seen in the water production rate. Schleicher et al. (1987) noted this in comparing the $\mathrm{OH}$ production rate variation with the visual light curve of Sekanina (1985). This general behavior, therefore, seems to be remarkably persistent from apparition to apparition.

\section{19P/BORRELLY}

Comet 19P/Borrelly is another short-period Jupiter family comet that was a flyby target of the New Millennium Program's Deep Space One spacecraft on 2001 September 22, when it became only the second comet after 1P/Halley to be imaged from nearby. It has a period of 6.85 years and a perihelion distance of $1.35 \mathrm{AU}$. Water production rates have been determined for 19P/Borrelly over several modern apparition, with maximum 
Table 1

Comet GZ: Observational Circumstances and Single-image Water Production Rates

\begin{tabular}{|c|c|c|c|c|c|}
\hline$\Delta T$ (days) & $r(\mathrm{AU})$ & $\Delta(\mathrm{AU})$ & $g\left(\mathrm{~s}^{-1}\right)$ & $Q\left(\mathrm{~s}^{-1}\right)$ & $\Delta Q\left(\mathrm{~s}^{-1}\right)$ \\
\hline \multicolumn{6}{|c|}{1998} \\
\hline-1.208 & 1.034 & 0.851 & 0.001764 & $5.68 \times 10^{28}$ & $2.1 \times 10^{26}$ \\
\hline 0.860 & 1.034 & 0.849 & 0.001766 & $4.82 \times 10^{28}$ & $2.5 \times 10^{26}$ \\
\hline 2.006 & 1.034 & 0.848 & 0.001767 & $5.49 \times 10^{28}$ & $3.1 \times 10^{27}$ \\
\hline 2.853 & 1.035 & 0.847 & 0.001768 & $4.71 \times 10^{28}$ & $1.3 \times 10^{26}$ \\
\hline 4.006 & 1.035 & 0.847 & 0.001766 & $4.46 \times 10^{28}$ & $4.1 \times 10^{25}$ \\
\hline 7.134 & 1.039 & 0.847 & 0.001767 & $4.38 \times 10^{28}$ & $1.3 \times 10^{26}$ \\
\hline 10.162 & 1.044 & 0.850 & 0.001768 & $3.91 \times 10^{28}$ & $3.3 \times 10^{26}$ \\
\hline 12.224 & 1.048 & 0.853 & 0.001769 & $4.54 \times 10^{28}$ & $4.3 \times 10^{26}$ \\
\hline 14.224 & 1.053 & 0.857 & 0.001770 & $4.50 \times 10^{28}$ & $2.2 \times 10^{26}$ \\
\hline 16.270 & 1.059 & 0.862 & 0.001773 & $3.95 \times 10^{28}$ & $5.0 \times 10^{25}$ \\
\hline 17.252 & 1.062 & 0.865 & 0.001774 & $4.18 \times 10^{28}$ & $1.7 \times 10^{26}$ \\
\hline 19.308 & 1.069 & 0.872 & 0.001776 & $3.21 \times 10^{28}$ & $2.0 \times 10^{26}$ \\
\hline 21.218 & 1.076 & 0.878 & 0.001778 & $3.47 \times 10^{28}$ & $2.0 \times 10^{26}$ \\
\hline 23.263 & 1.085 & 0.887 & 0.001781 & $2.88 \times 10^{28}$ & $6.6 \times 10^{25}$ \\
\hline 24.246 & 1.089 & 0.891 & 0.001782 & $2.86 \times 10^{28}$ & $2.4 \times 10^{26}$ \\
\hline 25.263 & 1.094 & 0.896 & 0.001783 & $2.68 \times 10^{28}$ & $7.0 \times 10^{25}$ \\
\hline 26.302 & 1.098 & 0.901 & 0.001787 & $3.18 \times 10^{28}$ & $2.1 \times 10^{26}$ \\
\hline 28.218 & 1.108 & 0.911 & 0.001789 & $2.92 \times 10^{28}$ & $2.4 \times 10^{26}$ \\
\hline \multicolumn{6}{|c|}{2005} \\
\hline-54.877 & 1.284 & 1.574 & 0.001450 & $3.49 \times 10^{28}$ & $3.7 \times 10^{26}$ \\
\hline-52.569 & 1.267 & 1.558 & 0.001450 & $3.98 \times 10^{28}$ & $3.1 \times 10^{26}$ \\
\hline-50.238 & 1.249 & 1.543 & 0.001439 & $3.95 \times 10^{28}$ & $3.3 \times 10^{26}$ \\
\hline-47.856 & 1.232 & 1.529 & 0.001439 & $3.09 \times 10^{28}$ & $3.7 \times 10^{26}$ \\
\hline-46.127 & 1.220 & 1.519 & 0.001439 & $3.33 \times 10^{28}$ & $3.3 \times 10^{26}$ \\
\hline-43.252 & 1.200 & 1.503 & 0.001431 & $4.18 \times 10^{28}$ & $3.0 \times 10^{26}$ \\
\hline-40.877 & 1.184 & 1.492 & 0.001431 & $4.88 \times 10^{28}$ & $2.7 \times 10^{26}$ \\
\hline-39.127 & 1.173 & 1.484 & 0.001422 & $4.92 \times 10^{28}$ & $2.4 \times 10^{26}$ \\
\hline-36.183 & 1.155 & 1.471 & 0.001422 & $4.60 \times 10^{28}$ & $2.6 \times 10^{26}$ \\
\hline-33.808 & 1.141 & 1.462 & 0.001414 & $6.69 \times 10^{28}$ & $1.8 \times 10^{26}$ \\
\hline-32.058 & 1.131 & 1.456 & 0.001414 & $5.64 \times 10^{28}$ & $2.0 \times 10^{26}$ \\
\hline-29.626 & 1.118 & 1.449 & 0.001407 & $4.49 \times 10^{28}$ & $2.6 \times 10^{26}$ \\
\hline-28.571 & 1.113 & 1.446 & 0.001407 & $4.56 \times 10^{28}$ & $2.3 \times 10^{26}$ \\
\hline-27.515 & 1.108 & 1.443 & 0.001407 & $5.57 \times 10^{28}$ & $2.0 \times 10^{26}$ \\
\hline-26.461 & 1.103 & 1.440 & 0.001407 & $6.13 \times 10^{28}$ & $1.7 \times 10^{26}$ \\
\hline-24.711 & 1.095 & 1.436 & 0.001401 & $6.69 \times 10^{28}$ & $1.8 \times 10^{26}$ \\
\hline-22.212 & 1.084 & 1.431 & 0.001395 & $5.72 \times 10^{28}$ & $1.9 \times 10^{26}$ \\
\hline-19.837 & 1.075 & 1.427 & 0.001395 & $5.90 \times 10^{28}$ & $2.1 \times 10^{26}$ \\
\hline-18.087 & 1.069 & 1.425 & 0.001389 & $6.96 \times 10^{28}$ & $1.8 \times 10^{26}$ \\
\hline-14.810 & 1.059 & 1.422 & 0.001383 & $6.75 \times 10^{28}$ & $2.6 \times 10^{26}$ \\
\hline-13.755 & 1.056 & 1.421 & 0.001383 & $6.56 \times 10^{28}$ & $2.8 \times 10^{26}$ \\
\hline-12.699 & 1.053 & 1.421 & 0.001383 & $6.40 \times 10^{28}$ & $2.7 \times 10^{26}$ \\
\hline-11.383 & 1.050 & 1.420 & 0.001379 & $5.99 \times 10^{28}$ & $2.5 \times 10^{26}$ \\
\hline-8.508 & 1.045 & 1.420 & 0.001375 & $4.39 \times 10^{28}$ & $3.7 \times 10^{26}$ \\
\hline-6.133 & 1.042 & 1.421 & 0.001375 & $5.89 \times 10^{28}$ & $2.7 \times 10^{26}$ \\
\hline-4.384 & 1.040 & 1.422 & 0.001372 & $3.64 \times 10^{28}$ & $3.4 \times 10^{26}$ \\
\hline-2.995 & 1.039 & 1.423 & 0.001372 & $4.28 \times 10^{28}$ & $3.0 \times 10^{26}$ \\
\hline-1.606 & 1.038 & 1.424 & 0.001370 & $3.07 \times 10^{28}$ & $1.4 \times 10^{27}$ \\
\hline-0.217 & 1.038 & 1.426 & 0.001370 & $4.29 \times 10^{28}$ & $3.1 \times 10^{27}$ \\
\hline 3.901 & 1.039 & 1.431 & 0.001367 & $3.12 \times 10^{28}$ & $3.7 \times 10^{27}$ \\
\hline 6.637 & 1.042 & 1.436 & 0.001366 & $4.26 \times 10^{28}$ & $2.0 \times 10^{26}$ \\
\hline 9.373 & 1.046 & 1.442 & 0.001366 & $3.94 \times 10^{28}$ & $2.0 \times 10^{26}$ \\
\hline 12.109 & 1.052 & 1.448 & 0.001366 & $3.14 \times 10^{28}$ & $3.0 \times 10^{26}$ \\
\hline 14.845 & 1.059 & 1.455 & 0.001365 & $2.64 \times 10^{28}$ & $3.7 \times 10^{27}$ \\
\hline 17.595 & 1.067 & 1.462 & 0.001366 & $2.88 \times 10^{28}$ & $3.3 \times 10^{26}$ \\
\hline 19.310 & 1.073 & 1.467 & 0.001367 & $2.43 \times 10^{28}$ & $4.1 \times 10^{26}$ \\
\hline 21.685 & 1.082 & 1.475 & 0.001367 & $1.55 \times 10^{28}$ & $1.8 \times 10^{27}$ \\
\hline 23.435 & 1.089 & 1.480 & 0.001368 & $1.70 \times 10^{28}$ & $1.2 \times 10^{26}$ \\
\hline 26.310 & 1.102 & 1.490 & 0.001371 & $1.33 \times 10^{28}$ & $8.3 \times 10^{26}$ \\
\hline
\end{tabular}

Notes.

$\Delta T$ : time from perihelion on 1998 November 21.3205 and 2005 July 2.7605 UT in days,

$r$ : heliocentric distance (AU),

$\Delta$ : geocentric distance (AU),

$g$ : solar Ly $\alpha$ g-factor (photons $\mathrm{s}^{-1}$ ),

$Q$ : water production rates for each image $\left(\mathrm{s}^{-1}\right)$,

$\Delta Q: 1 \sigma$ formal uncertainty $\left(\mathrm{s}^{-1}\right)$.

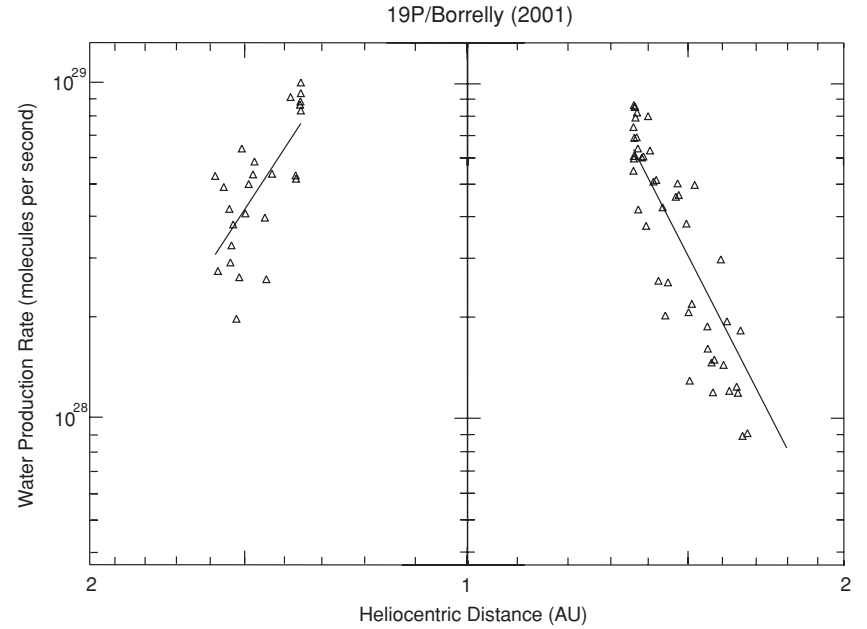

Figure 3. Single-image water production rates and fitted power-law distributions for comet 19P/Borrelly. The straight lines give the separate power-law variations pre- (left) and post-(right) perihelion of the form $2.8 \times 10^{29} r^{-5.2 \pm 1.1}$ and $3.5 \times$ $10^{29} r^{-6.6 \pm 0.6}$.

values near perihelion of 2.5-3.9 $\times 10^{28} \mathrm{~s}^{-1}$. (Bockelée-Morvan et al. 2002, 2004; Schleicher et al. 2003; Weaver et al. 2003; Fink 2009). 19P/Borrelly is normally classified as a $C_{2}$ depleted comet in the photometric (A'Hearn et al. 1995) and spectrophotometric (Fink 2009) surveys of comets whereas $\mathrm{CN}$ and $\mathrm{NH}_{2}$ are normal. In a study of data from multiple apparitions, including 2001, Schleicher et al. (2003) show that the water production rate is decidedly asymmetric about perihelion with the values being somewhat larger for comparable times before perihelion than after. After the Deep Space One images the nucleus of Borrelly is noteworthy for its highly triaxially shaped nucleus with one long axis and two shorter axes (Soderblom et al. 2002). The comet reached perihelion on September 14 during the 2001 apparition. $\mathrm{SOHO} / \mathrm{SWAN}$ obtained useful images of the hydrogen coma of 19P/Borrelly from 2001 July 4 through December 20, covering a range of heliocentric distances from 1.59 AU before perihelion to $1.80 \mathrm{AU}$ after perihelion.

Power-law fits of the production rate variation with heliocentric distance, $r$, are slightly asymmetric about perihelion with values of $2.8 \times 10^{29} r^{-5.2 \pm 1.1}$ and $3.5 \times 10^{29} r^{-6.6 \pm 0.6}$, pre- and post-perihelion, respectively, in molecules per second. Keep in mind that the power law is normalized by a fictitious production rate projected back to $1 \mathrm{AU}$, although the comet only has a perihelion distance of $1.35 \mathrm{AU}$, so the actual maximum production rate is never as large as the so-called $1 \mathrm{AU}$ value. The averaged power law over the whole apparition is then $3.3 \times$ $10^{29} r^{-6.1}$.

The single-image water production rate results are given in Figure 3 and Table 2. The water production rates over the apparition are very similar to the results of Bockelée-Morvan et al. (2002, 2004), Weaver et al. (2003), and Fink (2009) and are about a factor $40 \%$ larger than those from the ground-based $\mathrm{OH}$ observations of Schleicher et al. (2003), which is not unusual (Schleicher et al. 1998).

The SWAN observations of 19P/Borrelly were of sufficient quality that we could apply the deconvolution process in the TRM (Mäkinen \& Combi 2005) to extract daily average values of the water production rate at the nucleus for two extended time intervals: pre-perihelion and post-perihelion. The resulting water production rates are given in Table 3 . The single-image production rates are plotted as a function of time in Figure 4 
Table 2

Comet 19P/Borrelly: Observational Circumstances and Single-image Water Production Rates

\begin{tabular}{|c|c|c|c|c|c|}
\hline$\Delta T$ (days) & $r(\mathrm{AU})$ & $\Delta(\mathrm{AU})$ & $g\left(\mathrm{~s}^{-1}\right)$ & $Q\left(\mathrm{~s}^{-1}\right)$ & $\Delta Q\left(\mathrm{~s}^{-1}\right)$ \\
\hline-72.586 & 1.590 & 1.995 & 0.002134 & $4.05 \times 10^{28}$ & $1.0 \times 10^{27}$ \\
\hline-71.197 & 1.582 & 1.983 & 0.002134 & $2.24 \times 10^{28}$ & $1.4 \times 10^{27}$ \\
\hline-68.084 & 1.565 & 1.955 & 0.002133 & $3.78 \times 10^{28}$ & $7.5 \times 10^{26}$ \\
\hline-65.083 & 1.549 & 1.930 & 0.002131 & $3.30 \times 10^{28}$ & $8.9 \times 10^{26}$ \\
\hline-64.513 & 1.546 & 1.925 & 0.002131 & $2.37 \times 10^{28}$ & $2.3 \times 10^{26}$ \\
\hline-63.929 & 1.543 & 1.920 & 0.002131 & $2.63 \times 10^{28}$ & $2.1 \times 10^{26}$ \\
\hline-63.111 & 1.538 & 1.913 & 0.002120 & $3.00 \times 10^{28}$ & $9.4 \times 10^{26}$ \\
\hline-61.222 & 1.529 & 1.898 & 0.002119 & $1.67 \times 10^{28}$ & $1.3 \times 10^{27}$ \\
\hline-59.666 & 1.521 & 1.885 & 0.002118 & $2.16 \times 10^{28}$ & $2.5 \times 10^{26}$ \\
\hline-58.223 & 1.514 & 1.873 & 0.002118 & $4.80 \times 10^{28}$ & $7.3 \times 10^{26}$ \\
\hline-56.250 & 1.504 & 1.858 & 0.002117 & $3.21 \times 10^{28}$ & $9.3 \times 10^{26}$ \\
\hline-54.278 & 1.495 & 1.842 & 0.002116 & $3.85 \times 10^{28}$ & $6.8 \times 10^{26}$ \\
\hline-51.763 & 1.483 & 1.823 & 0.002105 & $4.09 \times 10^{28}$ & $1.5 \times 10^{26}$ \\
\hline-50.932 & 1.479 & 1.817 & 0.002104 & $4.43 \times 10^{28}$ & $5.7 \times 10^{26}$ \\
\hline-44.238 & 1.451 & 1.768 & 0.002092 & $3.12 \times 10^{28}$ & $8.8 \times 10^{26}$ \\
\hline-43.182 & 1.447 & 1.760 & 0.002091 & $2.13 \times 10^{28}$ & $1.2 \times 10^{27}$ \\
\hline-39.196 & 1.432 & 1.732 & 0.002089 & $4.10 \times 10^{28}$ & $8.2 \times 10^{26}$ \\
\hline-22.999 & 1.384 & 1.630 & 0.002058 & $6.61 \times 10^{28}$ & $9.5 \times 10^{25}$ \\
\hline-16.297 & 1.371 & 1.591 & 0.002049 & $4.06 \times 10^{28}$ & $6.1 \times 10^{26}$ \\
\hline-15.241 & 1.370 & 1.585 & 0.002048 & $3.98 \times 10^{28}$ & $6.4 \times 10^{26}$ \\
\hline-5.535 & 1.360 & 1.535 & 0.002035 & $6.30 \times 10^{28}$ & $3.4 \times 10^{26}$ \\
\hline-4.006 & 1.359 & 1.527 & 0.002034 & $6.43 \times 10^{28}$ & $7.1 \times 10^{25}$ \\
\hline-2.534 & 1.358 & 1.520 & 0.002033 & $6.77 \times 10^{28}$ & $3.6 \times 10^{26}$ \\
\hline-1.992 & 1.358 & 1.518 & 0.002033 & $6.08 \times 10^{28}$ & $7.8 \times 10^{25}$ \\
\hline-0.562 & 1.358 & 1.511 & 0.002032 & $7.24 \times 10^{28}$ & $3.4 \times 10^{26}$ \\
\hline 0.480 & 1.358 & 1.506 & 0.002032 & $5.48 \times 10^{28}$ & $8.3 \times 10^{25}$ \\
\hline 1.327 & 1.358 & 1.502 & 0.002031 & $4.18 \times 10^{28}$ & $5.1 \times 10^{26}$ \\
\hline 2.855 & 1.359 & 1.495 & 0.002031 & $4.58 \times 10^{28}$ & $9.6 \times 10^{25}$ \\
\hline 4.327 & 1.359 & 1.489 & 0.002030 & $6.29 \times 10^{28}$ & $3.5 \times 10^{26}$ \\
\hline 4.869 & 1.359 & 1.486 & 0.002030 & $4.51 \times 10^{28}$ & $9.0 \times 10^{25}$ \\
\hline 6.299 & 1.360 & 1.480 & 0.002026 & $5.13 \times 10^{28}$ & $3.9 \times 10^{26}$ \\
\hline 7.526 & 1.361 & 1.475 & 0.002025 & $6.22 \times 10^{28}$ & $7.2 \times 10^{25}$ \\
\hline 8.386 & 1.362 & 1.471 & 0.002024 & $8.60 \times 10^{28}$ & $2.5 \times 10^{26}$ \\
\hline 8.922 & 1.362 & 1.469 & 0.002024 & $6.22 \times 10^{28}$ & $7.0 \times 10^{25}$ \\
\hline 10.242 & 1.363 & 1.464 & 0.002024 & $5.82 \times 10^{28}$ & $8.7 \times 10^{25}$ \\
\hline 12.259 & 1.366 & 1.455 & 0.002021 & $5.15 \times 10^{28}$ & $1.0 \times 10^{26}$ \\
\hline 13.612 & 1.367 & 1.450 & 0.002020 & $6.00 \times 10^{28}$ & $3.2 \times 10^{26}$ \\
\hline 14.648 & 1.369 & 1.446 & 0.002020 & $4.80 \times 10^{28}$ & $9.2 \times 10^{25}$ \\
\hline 15.500 & 1.370 & 1.443 & 0.002019 & $3.29 \times 10^{28}$ & $4.9 \times 10^{26}$ \\
\hline 20.556 & 1.379 & 1.424 & 0.002015 & $4.54 \times 10^{28}$ & $3.6 \times 10^{26}$ \\
\hline 22.473 & 1.383 & 1.417 & 0.002014 & $4.57 \times 10^{28}$ & $3.5 \times 10^{26}$ \\
\hline 25.501 & 1.390 & 1.407 & 0.002012 & $2.97 \times 10^{28}$ & $6.0 \times 10^{26}$ \\
\hline 27.571 & 1.395 & 1.400 & 0.002010 & $5.87 \times 10^{28}$ & $2.9 \times 10^{26}$ \\
\hline 29.376 & 1.400 & 1.395 & 0.002009 & $4.74 \times 10^{28}$ & $3.3 \times 10^{26}$ \\
\hline 32.404 & 1.409 & 1.385 & 0.002009 & $3.91 \times 10^{28}$ & $4.2 \times 10^{26}$ \\
\hline 34.460 & 1.416 & 1.379 & 0.002007 & $3.95 \times 10^{28}$ & $4.4 \times 10^{26}$ \\
\hline 36.376 & 1.422 & 1.374 & 0.002006 & $2.11 \times 10^{28}$ & $7.3 \times 10^{26}$ \\
\hline 39.404 & 1.433 & 1.366 & 0.002006 & $3.33 \times 10^{28}$ & $5.4 \times 10^{26}$ \\
\hline 41.270 & 1.440 & 1.361 & 0.002005 & $1.70 \times 10^{28}$ & $8.0 \times 10^{26}$ \\
\hline 43.187 & 1.447 & 1.356 & 0.002004 & $2.09 \times 10^{28}$ & $7.2 \times 10^{26}$ \\
\hline 48.278 & 1.468 & 1.344 & 0.002003 & $3.56 \times 10^{28}$ & $5.0 \times 10^{26}$ \\
\hline 49.460 & 1.473 & 1.341 & 0.002002 & $3.87 \times 10^{28}$ & $8.4 \times 10^{25}$ \\
\hline 50.195 & 1.476 & 1.340 & 0.002002 & $3.60 \times 10^{28}$ & $4.6 \times 10^{26}$ \\
\hline 54.692 & 1.497 & 1.331 & 0.002003 & $3.01 \times 10^{28}$ & $5.4 \times 10^{26}$ \\
\hline 56.000 & 1.503 & 1.328 & 0.002003 & $1.74 \times 10^{28}$ & $1.9 \times 10^{26}$ \\
\hline 56.748 & 1.506 & 1.327 & 0.002002 & $1.14 \times 10^{28}$ & $1.3 \times 10^{27}$ \\
\hline 57.805 & 1.512 & 1.325 & 0.002001 & $1.83 \times 10^{28}$ & $9.5 \times 10^{26}$ \\
\hline 59.554 & 1.520 & 1.321 & 0.002000 & $3.83 \times 10^{28}$ & $3.8 \times 10^{26}$ \\
\hline 66.370 & 1.556 & 1.310 & 0.002000 & $1.59 \times 10^{28}$ & $2.0 \times 10^{26}$ \\
\hline 66.703 & 1.557 & 1.310 & 0.002000 & $1.39 \times 10^{28}$ & $2.2 \times 10^{26}$ \\
\hline 68.717 & 1.568 & 1.307 & 0.001999 & $1.27 \times 10^{28}$ & $2.4 \times 10^{26}$ \\
\hline 69.444 & 1.572 & 1.305 & 0.001999 & $1.06 \times 10^{28}$ & $1.5 \times 10^{27}$ \\
\hline 70.064 & 1.576 & 1.305 & 0.001999 & $1.29 \times 10^{28}$ & $3.1 \times 10^{26}$ \\
\hline 73.486 & 1.595 & 1.300 & 0.001996 & $2.41 \times 10^{28}$ & $6.7 \times 10^{26}$ \\
\hline 74.875 & 1.603 & 1.298 & 0.001995 & $1.25 \times 10^{28}$ & $1.1 \times 10^{27}$ \\
\hline 76.625 & 1.613 & 1.296 & 0.002001 & $1.64 \times 10^{28}$ & $8.2 \times 10^{26}$ \\
\hline
\end{tabular}

Table 2

(Continued)

\begin{tabular}{rccccc}
\hline \hline$\Delta T($ days $)$ & $r(\mathrm{AU})$ & $\Delta(\mathrm{AU})$ & $g\left(\mathrm{~s}^{-1}\right)$ & $Q\left(\mathrm{~s}^{-1}\right)$ & $\Delta Q\left(\mathrm{~s}^{-1}\right)$ \\
\hline 77.724 & 1.620 & 1.295 & 0.002001 & $1.07 \times 10^{28}$ & $2.9 \times 10^{26}$ \\
81.528 & 1.642 & 1.291 & 0.001998 & $1.09 \times 10^{28}$ & $1.5 \times 10^{27}$ \\
82.120 & 1.646 & 1.291 & 0.001998 & $1.05 \times 10^{28}$ & $2.9 \times 10^{26}$ \\
83.417 & 1.654 & 1.289 & 0.001997 & $1.55 \times 10^{28}$ & $8.9 \times 10^{26}$ \\
84.509 & 1.660 & 1.288 & 0.001996 & $8.05 \times 10^{27}$ & $3.8 \times 10^{26}$ \\
86.883 & 1.675 & 1.287 & 0.001995 & $8.19 \times 10^{27}$ & $3.6 \times 10^{26}$ \\
88.321 & 1.684 & 1.285 & 0.001994 & $1.12 \times 10^{28}$ & $1.3 \times 10^{27}$ \\
90.210 & 1.696 & 1.284 & 0.001993 & $1.45 \times 10^{28}$ & $1.0 \times 10^{27}$ \\
99.239 & 1.754 & 1.281 & 0.001996 & $1.91 \times 10^{28}$ & $7.0 \times 10^{26}$ \\
106.195 & 1.801 & 1.281 & 0.001992 & $2.44 \times 10^{28}$ & $6.2 \times 10^{26}$ \\
\hline
\end{tabular}

Notes.

$\Delta T$ : time from perihelion 2001 September 14.7055, in days for each SWAN image,

$r$ : heliocentric distance (AU),

$\Delta$ : geocentric distance (AU),

$g$ : solar Ly $\alpha$-factor (photons $\mathrm{s}^{-1}$ ),

$Q$ : water production rates for each image $\left(\mathrm{s}^{-1}\right)$,

$\Delta Q: 1 \sigma$ formal uncertainty $\left(\mathrm{s}^{-1}\right)$.

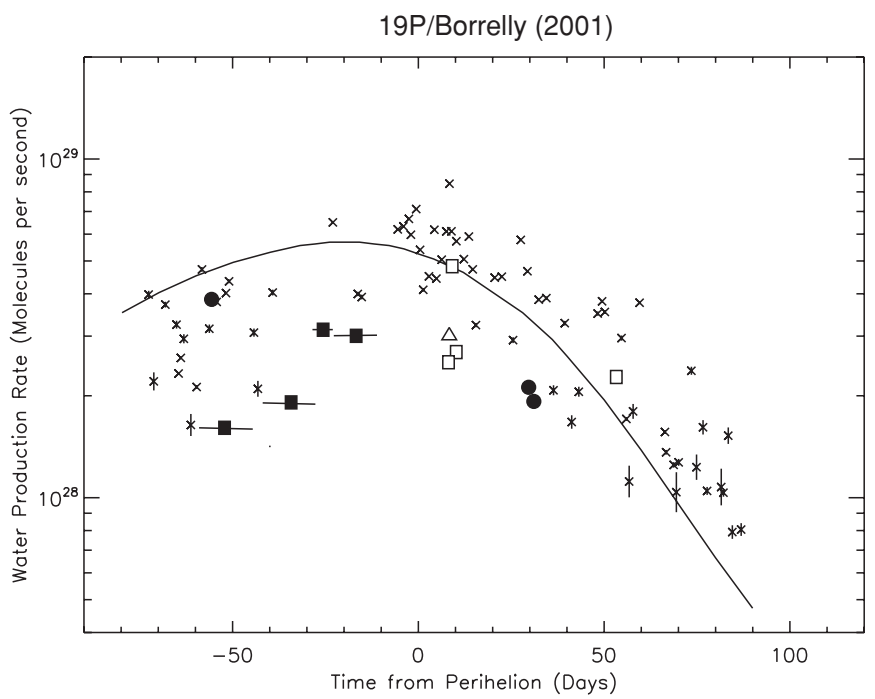

Figure 4. Single-image water production rates of comet 19P/Borrelly. The variation over the entire apparition from rough 70 days before perihelion to 80 days after perihelion is very similar to that found with the spin-axis, active area illumination model of Schleicher et al. (2003), shown as the solid curve rescaled upward by a factor of about two. Systematic differences by up to a factor of about 2.5 between water production rates determined from groundbased photometry of $\mathrm{OH}$ and $\mathrm{H}$ Ly $\alpha$ observations by SWAN are not unusual (Combi et al. 2000, 2005). A number of other water production rates are shown. The filled squares are from Bockelée-Morvan et al. (2002), open squares from Bockelée-Morvan et al. (2004), the diamond from Weaver et al. (2003), and the filled circles from Fink (2009). The horizontal lines from Bockelée-Morvan et al. (2002) indicated several day to two-week integrations.

and compared with a number of other measures of the water production rate. Except for an overall scaling factor, mentioned above, the shape of the variation over the whole apparition is very similar to that from the polar active-area solar-illumination model of Schleicher et al. (2003), which explains the general long-term trend in production rate as being due to seasonal solar illumination of active areas on the nucleus. The scaled model of Schleicher et al. is plotted as the solid line in Figure 4.

\section{81P/WILD 2}

Comet $81 \mathrm{P} /$ Wild 2 is another short-period Jupiter family comet with an interesting history. It was discovered in 1978 after gravitational interaction with Jupiter in 1974 brought it 
Table 3

Comet 19P/Borrelly: Deconvolved Daily Average Water Production Rates

\begin{tabular}{|c|c|c|}
\hline$\Delta T$ (days) & $Q\left(\mathrm{~s}^{-1}\right)$ & $\Delta Q\left(\mathrm{~s}^{-1}\right)$ \\
\hline-71.706 & $3.28 \times 10^{28}$ & $3.2 \times 10^{28}$ \\
\hline-70.706 & $3.37 \times 10^{28}$ & $3.0 \times 10^{28}$ \\
\hline-69.706 & $3.45 \times 10^{28}$ & $2.6 \times 10^{28}$ \\
\hline-68.706 & $3.24 \times 10^{28}$ & $3.0 \times 10^{28}$ \\
\hline-67.706 & $3.06 \times 10^{28}$ & $3.0 \times 10^{28}$ \\
\hline-66.706 & $3.12 \times 10^{28}$ & $2.6 \times 10^{28}$ \\
\hline-65.706 & $2.96 \times 10^{28}$ & $3.4 \times 10^{28}$ \\
\hline-64.706 & $3.22 \times 10^{28}$ & $3.4 \times 10^{28}$ \\
\hline-63.706 & $3.56 \times 10^{28}$ & $2.9 \times 10^{28}$ \\
\hline-62.706 & $3.08 \times 10^{28}$ & $3.0 \times 10^{28}$ \\
\hline-61.706 & $3.15 \times 10^{28}$ & $3.1 \times 10^{28}$ \\
\hline-60.706 & $3.20 \times 10^{28}$ & $2.8 \times 10^{28}$ \\
\hline-59.706 & $3.24 \times 10^{28}$ & $2.9 \times 10^{28}$ \\
\hline-58.706 & $3.43 \times 10^{28}$ & $2.5 \times 10^{28}$ \\
\hline-57.706 & $3.71 \times 10^{28}$ & $2.2 \times 10^{28}$ \\
\hline-56.706 & $3.77 \times 10^{28}$ & $2.5 \times 10^{28}$ \\
\hline-55.706 & $4.17 \times 10^{28}$ & $2.2 \times 10^{28}$ \\
\hline-54.706 & $4.54 \times 10^{28}$ & $1.4 \times 10^{28}$ \\
\hline-53.706 & $3.92 \times 10^{28}$ & $2.8 \times 10^{28}$ \\
\hline-52.706 & $3.97 \times 10^{28}$ & $2.7 \times 10^{28}$ \\
\hline-51.706 & $3.93 \times 10^{28}$ & $2.5 \times 10^{28}$ \\
\hline-50.706 & $3.56 \times 10^{28}$ & $2.4 \times 10^{28}$ \\
\hline-49.706 & $3.31 \times 10^{28}$ & $4.9 \times 10^{28}$ \\
\hline-48.706 & $3.54 \times 10^{28}$ & $4.2 \times 10^{28}$ \\
\hline-47.706 & $3.30 \times 10^{28}$ & $5.4 \times 10^{28}$ \\
\hline-46.706 & $3.51 \times 10^{28}$ & $5.2 \times 10^{28}$ \\
\hline-45.706 & $3.28 \times 10^{28}$ & $5.9 \times 10^{28}$ \\
\hline-44.706 & $3.39 \times 10^{28}$ & $5.9 \times 10^{28}$ \\
\hline-43.706 & $3.06 \times 10^{28}$ & $4.6 \times 10^{28}$ \\
\hline-42.706 & $3.66 \times 10^{28}$ & $3.9 \times 10^{28}$ \\
\hline-39.706 & $3.68 \times 10^{28}$ & $3.8 \times 10^{28}$ \\
\hline-38.706 & $4.03 \times 10^{28}$ & $3.7 \times 10^{28}$ \\
\hline-37.706 & $4.42 \times 10^{28}$ & $3.3 \times 10^{28}$ \\
\hline-36.706 & $6.17 \times 10^{28}$ & $3.1 \times 10^{28}$ \\
\hline-35.706 & $7.14 \times 10^{28}$ & $3.8 \times 10^{28}$ \\
\hline-34.706 & $8.27 \times 10^{28}$ & $5.0 \times 10^{28}$ \\
\hline-33.706 & $9.39 \times 10^{28}$ & $6.6 \times 10^{28}$ \\
\hline-32.706 & $5.06 \times 10^{28}$ & $2.3 \times 10^{28}$ \\
\hline-31.706 & $5.22 \times 10^{28}$ & $2.8 \times 10^{28}$ \\
\hline-30.706 & $5.36 \times 10^{28}$ & $2.7 \times 10^{28}$ \\
\hline-29.706 & $5.51 \times 10^{28}$ & $2.7 \times 10^{28}$ \\
\hline-28.706 & $5.60 \times 10^{28}$ & $2.4 \times 10^{28}$ \\
\hline-27.706 & $5.28 \times 10^{28}$ & $2.9 \times 10^{28}$ \\
\hline-26.706 & $5.86 \times 10^{28}$ & $1.7 \times 10^{28}$ \\
\hline-25.706 & $7.88 \times 10^{28}$ & $4.7 \times 10^{28}$ \\
\hline-24.706 & $7.42 \times 10^{28}$ & $4.2 \times 10^{28}$ \\
\hline-23.706 & $6.84 \times 10^{28}$ & $3.6 \times 10^{28}$ \\
\hline-22.706 & $6.47 \times 10^{28}$ & $3.3 \times 10^{28}$ \\
\hline-21.706 & $5.72 \times 10^{28}$ & $3.5 \times 10^{28}$ \\
\hline-20.706 & $5.55 \times 10^{28}$ & $3.3 \times 10^{28}$ \\
\hline-19.706 & $5.35 \times 10^{28}$ & $3.5 \times 10^{28}$ \\
\hline-18.706 & $5.24 \times 10^{28}$ & $3.1 \times 10^{28}$ \\
\hline-17.706 & $5.12 \times 10^{28}$ & $3.9 \times 10^{28}$ \\
\hline-16.706 & $5.04 \times 10^{28}$ & $3.8 \times 10^{28}$ \\
\hline-15.706 & $4.86 \times 10^{28}$ & $1.1 \times 10^{29}$ \\
\hline-14.706 & $4.91 \times 10^{28}$ & $1.0 \times 10^{29}$ \\
\hline-13.706 & $4.90 \times 10^{28}$ & $6.0 \times 10^{28}$ \\
\hline-12.706 & $5.01 \times 10^{28}$ & $5.4 \times 10^{28}$ \\
\hline-11.706 & $5.52 \times 10^{28}$ & $4.0 \times 10^{28}$ \\
\hline-10.706 & $5.67 \times 10^{28}$ & $3.3 \times 10^{28}$ \\
\hline-9.706 & $5.88 \times 10^{28}$ & $2.1 \times 10^{28}$ \\
\hline-8.706 & $6.00 \times 10^{28}$ & $1.4 \times 10^{28}$ \\
\hline-7.706 & $6.05 \times 10^{28}$ & $1.1 \times 10^{28}$ \\
\hline-6.706 & $6.02 \times 10^{28}$ & $4.4 \times 10^{27}$ \\
\hline-5.706 & $6.52 \times 10^{28}$ & $2.0 \times 10^{28}$ \\
\hline-4.706 & $6.91 \times 10^{28}$ & $1.2 \times 10^{28}$ \\
\hline
\end{tabular}

Table 3

(Continued)

\begin{tabular}{|c|c|c|}
\hline$\Delta T$ (days) & $Q\left(\mathrm{~s}^{-1}\right)$ & $\Delta Q\left(\mathrm{~s}^{-1}\right)$ \\
\hline-3.706 & $5.98 \times 10^{28}$ & $2.5 \times 10^{28}$ \\
\hline-2.706 & $5.83 \times 10^{28}$ & $1.9 \times 10^{28}$ \\
\hline-1.706 & $6.04 \times 10^{28}$ & $2.4 \times 10^{28}$ \\
\hline-0.706 & $5.98 \times 10^{28}$ & $2.0 \times 10^{28}$ \\
\hline 0.294 & $6.23 \times 10^{28}$ & $2.1 \times 10^{28}$ \\
\hline 1.294 & $6.22 \times 10^{28}$ & $1.6 \times 10^{28}$ \\
\hline 2.294 & $6.37 \times 10^{28}$ & $2.0 \times 10^{28}$ \\
\hline 3.294 & $6.50 \times 10^{28}$ & $1.5 \times 10^{28}$ \\
\hline 4.294 & $6.25 \times 10^{28}$ & $1.7 \times 10^{28}$ \\
\hline 5.294 & $6.63 \times 10^{28}$ & $1.1 \times 10^{28}$ \\
\hline 6.294 & $5.68 \times 10^{28}$ & $1.6 \times 10^{28}$ \\
\hline 7.294 & $5.86 \times 10^{28}$ & $8.3 \times 10^{27}$ \\
\hline 8.294 & $5.24 \times 10^{28}$ & $1.3 \times 10^{28}$ \\
\hline 9.294 & $5.14 \times 10^{28}$ & $7.3 \times 10^{27}$ \\
\hline 10.294 & $5.35 \times 10^{28}$ & $3.5 \times 10^{28}$ \\
\hline 11.294 & $5.29 \times 10^{28}$ & $2.6 \times 10^{28}$ \\
\hline 12.294 & $5.06 \times 10^{28}$ & $4.7 \times 10^{28}$ \\
\hline 13.294 & $4.97 \times 10^{28}$ & $4.6 \times 10^{28}$ \\
\hline 14.294 & $5.25 \times 10^{28}$ & $3.5 \times 10^{28}$ \\
\hline 15.294 & $5.22 \times 10^{28}$ & $3.2 \times 10^{28}$ \\
\hline 16.294 & $5.19 \times 10^{28}$ & $2.5 \times 10^{28}$ \\
\hline 17.294 & $5.36 \times 10^{28}$ & $1.8 \times 10^{28}$ \\
\hline 18.294 & $5.22 \times 10^{28}$ & $2.6 \times 10^{28}$ \\
\hline 19.294 & $5.31 \times 10^{28}$ & $2.0 \times 10^{28}$ \\
\hline 20.294 & $4.84 \times 10^{28}$ & $3.0 \times 10^{28}$ \\
\hline 21.294 & $5.13 \times 10^{28}$ & $2.7 \times 10^{28}$ \\
\hline 22.294 & $5.08 \times 10^{28}$ & $2.7 \times 10^{28}$ \\
\hline 23.294 & $5.56 \times 10^{28}$ & $2.3 \times 10^{28}$ \\
\hline 24.294 & $4.69 \times 10^{28}$ & $2.8 \times 10^{28}$ \\
\hline 25.294 & $4.96 \times 10^{28}$ & $2.4 \times 10^{28}$ \\
\hline 26.294 & $4.24 \times 10^{28}$ & $3.1 \times 10^{28}$ \\
\hline 27.294 & $4.32 \times 10^{28}$ & $2.8 \times 10^{28}$ \\
\hline 28.294 & $4.37 \times 10^{28}$ & $2.8 \times 10^{28}$ \\
\hline 29.294 & $4.58 \times 10^{28}$ & $2.1 \times 10^{28}$ \\
\hline 30.294 & $3.99 \times 10^{28}$ & $2.5 \times 10^{28}$ \\
\hline 31.294 & $4.21 \times 10^{28}$ & $1.8 \times 10^{28}$ \\
\hline 32.294 & $3.49 \times 10^{28}$ & $3.5 \times 10^{28}$ \\
\hline 33.294 & $3.77 \times 10^{28}$ & $2.6 \times 10^{28}$ \\
\hline 34.294 & $3.55 \times 10^{28}$ & $2.4 \times 10^{28}$ \\
\hline 35.294 & $3.54 \times 10^{28}$ & $2.0 \times 10^{28}$ \\
\hline 36.294 & $3.03 \times 10^{28}$ & $2.7 \times 10^{28}$ \\
\hline 37.294 & $2.92 \times 10^{28}$ & $2.2 \times 10^{28}$ \\
\hline 38.294 & $3.12 \times 10^{28}$ & $3.1 \times 10^{28}$ \\
\hline 39.294 & $3.27 \times 10^{28}$ & $2.7 \times 10^{28}$ \\
\hline 40.294 & $2.90 \times 10^{28}$ & $3.2 \times 10^{28}$ \\
\hline 41.294 & $3.23 \times 10^{28}$ & $2.9 \times 10^{28}$ \\
\hline 42.294 & $3.25 \times 10^{28}$ & $2.5 \times 10^{28}$ \\
\hline 43.294 & $3.51 \times 10^{28}$ & $2.1 \times 10^{28}$ \\
\hline 44.294 & $3.81 \times 10^{28}$ & $1.6 \times 10^{28}$ \\
\hline 45.294 & $4.20 \times 10^{28}$ & $1.1 \times 10^{28}$ \\
\hline 46.294 & $3.53 \times 10^{28}$ & $2.0 \times 10^{28}$ \\
\hline 47.294 & $3.99 \times 10^{28}$ & $1.4 \times 10^{28}$ \\
\hline 48.294 & $2.99 \times 10^{28}$ & $2.0 \times 10^{28}$ \\
\hline 49.294 & $3.09 \times 10^{28}$ & $1.9 \times 10^{28}$ \\
\hline 50.294 & $3.28 \times 10^{28}$ & $1.6 \times 10^{28}$ \\
\hline 51.294 & $3.24 \times 10^{28}$ & $1.2 \times 10^{28}$ \\
\hline 52.294 & $2.46 \times 10^{28}$ & $1.1 \times 10^{28}$ \\
\hline 53.294 & $2.41 \times 10^{28}$ & $7.5 \times 10^{27}$ \\
\hline 54.294 & $2.56 \times 10^{28}$ & $2.9 \times 10^{28}$ \\
\hline 55.294 & $2.77 \times 10^{28}$ & $2.8 \times 10^{28}$ \\
\hline 56.294 & $1.84 \times 10^{28}$ & $2.2 \times 10^{28}$ \\
\hline 57.294 & $1.81 \times 10^{28}$ & $2.3 \times 10^{28}$ \\
\hline 58.294 & $1.88 \times 10^{28}$ & $2.0 \times 10^{28}$ \\
\hline 59.294 & $1.97 \times 10^{28}$ & $1.9 \times 10^{28}$ \\
\hline 60.294 & $2.03 \times 10^{28}$ & $3.3 \times 10^{28}$ \\
\hline 61.294 & $2.12 \times 10^{28}$ & $2.8 \times 10^{28}$ \\
\hline
\end{tabular}


Table 3

(Continued)

\begin{tabular}{|c|c|c|}
\hline$\Delta T$ (days) & $Q\left(\mathrm{~s}^{-1}\right)$ & $\Delta Q\left(\mathrm{~s}^{-1}\right)$ \\
\hline 62.294 & $2.22 \times 10^{28}$ & $2.5 \times 10^{28}$ \\
\hline 63.294 & $2.20 \times 10^{28}$ & $2.1 \times 10^{28}$ \\
\hline 64.294 & $1.98 \times 10^{28}$ & $2.7 \times 10^{28}$ \\
\hline 65.294 & $2.09 \times 10^{28}$ & $1.5 \times 10^{28}$ \\
\hline 66.294 & $2.01 \times 10^{28}$ & $1.6 \times 10^{28}$ \\
\hline 67.294 & $1.99 \times 10^{28}$ & $1.4 \times 10^{28}$ \\
\hline 68.294 & $1.93 \times 10^{28}$ & $2.7 \times 10^{28}$ \\
\hline 69.294 & $2.24 \times 10^{28}$ & $2.4 \times 10^{28}$ \\
\hline 70.294 & $2.04 \times 10^{28}$ & $1.7 \times 10^{28}$ \\
\hline 71.294 & $2.37 \times 10^{28}$ & $1.5 \times 10^{28}$ \\
\hline 72.294 & $2.67 \times 10^{28}$ & $1.8 \times 10^{28}$ \\
\hline 73.294 & $3.11 \times 10^{28}$ & $1.5 \times 10^{28}$ \\
\hline 74.294 & $1.53 \times 10^{28}$ & $1.6 \times 10^{28}$ \\
\hline 75.294 & $1.42 \times 10^{28}$ & $1.5 \times 10^{28}$ \\
\hline 76.294 & $1.46 \times 10^{28}$ & $1.7 \times 10^{28}$ \\
\hline 77.294 & $1.63 \times 10^{28}$ & $1.4 \times 10^{28}$ \\
\hline 78.294 & $1.61 \times 10^{28}$ & $1.5 \times 10^{28}$ \\
\hline 79.294 & $1.66 \times 10^{28}$ & $1.2 \times 10^{28}$ \\
\hline 80.294 & $1.45 \times 10^{28}$ & $1.4 \times 10^{28}$ \\
\hline 81.294 & $1.43 \times 10^{28}$ & $1.1 \times 10^{28}$ \\
\hline 82.294 & $1.53 \times 10^{28}$ & $1.1 \times 10^{28}$ \\
\hline 83.294 & $1.65 \times 10^{28}$ & $1.0 \times 10^{28}$ \\
\hline 84.294 & $1.85 \times 10^{28}$ & $9.1 \times 10^{27}$ \\
\hline 85.294 & $2.09 \times 10^{28}$ & $7.0 \times 10^{27}$ \\
\hline 86.294 & $1.65 \times 10^{28}$ & $2.6 \times 10^{28}$ \\
\hline 87.294 & $2.04 \times 10^{28}$ & $1.8 \times 10^{28}$ \\
\hline 88.294 & $1.41 \times 10^{28}$ & $3.9 \times 10^{28}$ \\
\hline 89.294 & $1.46 \times 10^{28}$ & $4.2 \times 10^{28}$ \\
\hline 90.294 & $1.50 \times 10^{28}$ & $3.7 \times 10^{28}$ \\
\hline 91.294 & $1.85 \times 10^{28}$ & $2.4 \times 10^{28}$ \\
\hline 92.294 & $1.74 \times 10^{28}$ & $2.4 \times 10^{28}$ \\
\hline 93.294 & $1.96 \times 10^{28}$ & $2.3 \times 10^{28}$ \\
\hline 94.294 & $2.13 \times 10^{28}$ & $1.8 \times 10^{28}$ \\
\hline 95.294 & $2.68 \times 10^{28}$ & $1.5 \times 10^{28}$ \\
\hline
\end{tabular}

Notes.

$\Delta T$ : time from perihelion 2001 September 14.7055 , in days for each deconvolved value,

$Q$ : water production rates for each image $\left(\mathrm{s}^{-1}\right)$,

$\Delta Q: 1 \sigma$ formal uncertainty $\left(\mathrm{s}^{-1}\right)$.

into an orbit with a perihelion distance of only 1.5 AU (Sekanina $\&$ Yeomans 1985). It has been on the short list of virtually every comet mission ever conceived for the last 25 years. It was the target of the Stardust mission (Brownlee et al. 2004) and therefore is the only object, besides the Moon, from which material has been returned to Earth by a spacecraft for laboratory analysis.

An analysis of SWAN observations of 81P/Wild 2 during the 1997 apparition was included in the paper by Mäkinen et al. (2001), but this was before the analysis procedure was improved with the TRM (Mäkinen \& Combi 2005) that also includes better lifetime and exothermic (i.e., vectorial) velocity information for daughter species than the previous simple Haser analysis. Clearly, SWAN was operating during the 2003-2004 apparition that included the Stardust flyby; however, the comet spent all of its active period being occulted by portions of the $\mathrm{SOHO}$ spacecraft itself, so there were no useful observations. Furthermore, the 81P/Wild 2 observations of 1997 were of sufficiently high quality that we could also apply the full temporal deconvolution procedure for calculating daily average values of the water production rate at the nucleus. Table 4 gives the results for the single-image values of the production rates
Table 4

Comet 81P/Wild 2: Observational Circumstances and Single-image Water Production Rates

\begin{tabular}{|c|c|c|c|c|c|}
\hline$\Delta T$ (days) & $r(\mathrm{AU})$ & $\Delta(\mathrm{AU})$ & $g\left(\mathrm{~s}^{-1}\right)$ & $Q\left(\mathrm{~s}^{-1}\right)$ & $\Delta Q\left(\mathrm{~s}^{-1}\right)$ \\
\hline-37.071 & 1.626 & 0.980 & 0.001424 & $2.40 \times 10^{28}$ & $6.4 \times 10^{25}$ \\
\hline-32.328 & 1.615 & 1.002 & 0.001425 & $3.44 \times 10^{28}$ & $3.2 \times 10^{25}$ \\
\hline-30.286 & 1.611 & 1.012 & 0.001426 & $3.65 \times 10^{28}$ & $3.2 \times 10^{25}$ \\
\hline-27.605 & 1.607 & 1.025 & 0.001422 & $3.01 \times 10^{28}$ & $4.0 \times 10^{25}$ \\
\hline-25.439 & 1.603 & 1.036 & 0.001423 & $3.42 \times 10^{28}$ & $3.1 \times 10^{25}$ \\
\hline-24.360 & 1.601 & 1.041 & 0.001423 & $3.65 \times 10^{28}$ & $1.1 \times 10^{25}$ \\
\hline-23.319 & 1.600 & 1.047 & 0.001423 & $4.01 \times 10^{28}$ & $2.5 \times 10^{25}$ \\
\hline-21.927 & 1.598 & 1.054 & 0.001424 & $4.70 \times 10^{28}$ & $8.8 \times 10^{24}$ \\
\hline-20.620 & 1.596 & 1.061 & 0.001424 & $5.39 \times 10^{28}$ & $2.3 \times 10^{25}$ \\
\hline-19.440 & 1.595 & 1.067 & 0.001425 & $5.13 \times 10^{28}$ & $8.2 \times 10^{24}$ \\
\hline-18.453 & 1.593 & 1.072 & 0.001425 & $4.44 \times 10^{28}$ & $2.4 \times 10^{25}$ \\
\hline-17.270 & 1.592 & 1.079 & 0.001422 & $3.41 \times 10^{28}$ & $1.2 \times 10^{25}$ \\
\hline-16.301 & 1.591 & 1.084 & 0.001422 & $3.96 \times 10^{28}$ & $3.1 \times 10^{25}$ \\
\hline-14.854 & 1.590 & 1.092 & 0.001422 & $3.05 \times 10^{28}$ & $1.3 \times 10^{25}$ \\
\hline-13.620 & 1.589 & 1.099 & 0.001423 & $3.09 \times 10^{28}$ & $3.9 \times 10^{25}$ \\
\hline-12.423 & 1.588 & 1.106 & 0.001423 & $2.74 \times 10^{28}$ & $1.7 \times 10^{25}$ \\
\hline-10.271 & 1.586 & 1.118 & 0.001424 & $1.90 \times 10^{28}$ & $2.5 \times 10^{25}$ \\
\hline-9.315 & 1.585 & 1.124 & 0.001424 & $2.66 \times 10^{28}$ & $4.8 \times 10^{25}$ \\
\hline-7.854 & 1.585 & 1.132 & 0.001422 & $1.63 \times 10^{28}$ & $2.9 \times 10^{25}$ \\
\hline-4.468 & 1.583 & 1.152 & 0.001424 & $2.10 \times 10^{28}$ & $6.0 \times 10^{25}$ \\
\hline-2.514 & 1.583 & 1.164 & 0.001424 & $2.17 \times 10^{28}$ & $6.0 \times 10^{25}$ \\
\hline 0.490 & 1.583 & 1.183 & 0.001425 & $1.44 \times 10^{28}$ & $7.7 \times 10^{25}$ \\
\hline 4.671 & 1.583 & 1.209 & 0.001427 & $2.00 \times 10^{28}$ & $6.1 \times 10^{25}$ \\
\hline 7.163 & 1.584 & 1.226 & 0.001427 & $1.56 \times 10^{28}$ & $8.4 \times 10^{25}$ \\
\hline 9.219 & 1.585 & 1.240 & 0.001426 & $2.60 \times 10^{28}$ & $5.3 \times 10^{25}$ \\
\hline 10.969 & 1.586 & 1.251 & 0.001427 & $2.05 \times 10^{28}$ & $7.3 \times 10^{25}$ \\
\hline 17.955 & 1.593 & 1.300 & 0.001429 & $1.94 \times 10^{28}$ & $9.5 \times 10^{25}$ \\
\hline 25.093 & 1.603 & 1.353 & 0.001430 & $2.22 \times 10^{28}$ & $8.3 \times 10^{25}$ \\
\hline 29.043 & 1.609 & 1.384 & 0.001431 & $1.25 \times 10^{28}$ & $1.4 \times 10^{26}$ \\
\hline 35.496 & 1.622 & 1.436 & 0.001433 & $1.13 \times 10^{28}$ & $1.6 \times 10^{26}$ \\
\hline 38.100 & 1.628 & 1.458 & 0.001434 & $1.28 \times 10^{28}$ & $1.4 \times 10^{26}$ \\
\hline 42.835 & 1.640 & 1.499 & 0.001435 & $1.65 \times 10^{28}$ & $9.9 \times 10^{25}$ \\
\hline 53.649 & 1.670 & 1.598 & 0.001440 & $9.18 \times 10^{27}$ & $1.9 \times 10^{26}$ \\
\hline
\end{tabular}

Notes.

$\Delta T$ : time from perihelion 1997 May 6.6272, in days for each SWAN image,

$r$ : heliocentric distance (AU),

$\Delta$ : geocentric distance (AU),

$g$ : solar Ly $\alpha$-factor (photons $\mathrm{s}^{-1}$ ),

$Q$ : water production rates for each image $\left(\mathrm{s}^{-1}\right)$,

$\Delta Q: 1 \sigma$ formal uncertainty $\left(\mathrm{s}^{-1}\right)$.

from the SWAN images of 81P/Wild 2 and the observational circumstances. These results are similar to, but not exactly the same as, those published earlier by Mäkinen et al. (2001) because of the model improvements just mentioned.

The daily average values obtained with the TRM deconvolution procedure are given in Table 5 and plotted as a function of time in Figure 5. The rise in production rate to the maximum around 25 days before perihelion, as would be expected, happens a few days earlier in the daily average values than in the single-image values, since it is correctly accounting for the slower response of filling the coma with $\mathrm{H}$ atoms after a rise in water production rate at the nucleus. The filling of the coma after an outburst was observed by SWAN in the case of Comet 1996 B2 (Hyakutake), because of the proximity of the comet (Bertaux et al. 1998). The single-image values represent then the response of the whole hydrogen coma to changes in the water production rate. The combination of the 1-2 day production time from photodissociation of water and $\mathrm{OH}$, as well as the filling time of the large FOV seen by SWAN cause the delay in the single-image production rates. 
Table 5

Comet 81P/Wild 2: Deconvolved Daily Average Water Production Rates

\begin{tabular}{|c|c|c|}
\hline$\Delta T$ (days) & $Q\left(\mathrm{~s}^{-1}\right)$ & $\Delta Q\left(\mathrm{~s}^{-1}\right)$ \\
\hline-44.627 & $1.59 \times 10^{28}$ & $1.4 \times 10^{28}$ \\
\hline-43.627 & $1.79 \times 10^{28}$ & $2.4 \times 10^{28}$ \\
\hline-42.627 & $2.01 \times 10^{28}$ & $2.2 \times 10^{28}$ \\
\hline-41.627 & $2.35 \times 10^{28}$ & $1.8 \times 10^{28}$ \\
\hline-40.627 & $2.88 \times 10^{28}$ & $1.4 \times 10^{28}$ \\
\hline-39.627 & $3.36 \times 10^{28}$ & $1.4 \times 10^{28}$ \\
\hline-38.627 & $2.77 \times 10^{28}$ & $1.9 \times 10^{28}$ \\
\hline-37.627 & $2.90 \times 10^{28}$ & $2.7 \times 10^{28}$ \\
\hline-36.627 & $3.26 \times 10^{28}$ & $2.4 \times 10^{28}$ \\
\hline-35.627 & $3.56 \times 10^{28}$ & $1.9 \times 10^{28}$ \\
\hline-34.627 & $3.91 \times 10^{28}$ & $1.3 \times 10^{2}$ \\
\hline-33.627 & $3.73 \times 10^{28}$ & $1.5 \times 10^{28}$ \\
\hline-32.627 & $3.91 \times 10^{28}$ & $1.2 \times 10^{28}$ \\
\hline-31.627 & $3.63 \times 10^{28}$ & $1.8 \times 10^{28}$ \\
\hline-30.627 & $3.77 \times 10^{28}$ & $1.5 \times 10^{28}$ \\
\hline-29.627 & $3.93 \times 10^{28}$ & $1.7 \times 10^{28}$ \\
\hline-28.627 & $4.03 \times 10^{28}$ & $1.5 \times 10^{28}$ \\
\hline-27.627 & $4.27 \times 10^{28}$ & $1.9 \times 10^{28}$ \\
\hline-26.627 & $4.46 \times 10^{28}$ & $1.6 \times 10^{28}$ \\
\hline-25.627 & $4.66 \times 10^{28}$ & $2.3 \times 10^{28}$ \\
\hline-24.627 & $4.97 \times 10^{28}$ & $1.9 \times 10^{28}$ \\
\hline-23.627 & $4.05 \times 10^{28}$ & $2.6 \times 10^{2}$ \\
\hline-22.627 & $4.47 \times 10^{28}$ & $2.2 \times 10^{28}$ \\
\hline-21.627 & $3.96 \times 10^{28}$ & $2.0 \times 10^{28}$ \\
\hline-20.627 & $4.14 \times 10^{28}$ & $1.6 \times 10^{28}$ \\
\hline-19.627 & $3.66 \times 10^{28}$ & $2.0 \times 10^{28}$ \\
\hline-18.627 & $3.76 \times 10^{28}$ & $1.6 \times 10^{28}$ \\
\hline-17.627 & $3.38 \times 10^{28}$ & $2.2 \times 10^{28}$ \\
\hline-16.627 & $3.23 \times 10^{28}$ & $1.8 \times 10^{28}$ \\
\hline-15.627 & $3.11 \times 10^{28}$ & $1.5 \times 10^{28}$ \\
\hline-14.627 & $2.98 \times 10^{28}$ & $1.1 \times 10^{28}$ \\
\hline-13.627 & $2.61 \times 10^{28}$ & $1.0 \times 10^{28}$ \\
\hline-12.627 & $2.43 \times 10^{28}$ & $6.9 \times 10^{2}$ \\
\hline-11.627 & $2.48 \times 10^{28}$ & $1.2 \times 10^{28}$ \\
\hline-10.627 & $2.34 \times 10^{28}$ & $9.1 \times 10^{2}$ \\
\hline-9.627 & $2.56 \times 10^{28}$ & $1.8 \times 10^{28}$ \\
\hline-8.627 & $2.51 \times 10^{28}$ & $1.6 \times 10^{28}$ \\
\hline-7.627 & $2.40 \times 10^{28}$ & $1.6 \times 10^{28}$ \\
\hline-6.627 & $2.37 \times 10^{28}$ & $1.5 \times 10^{28}$ \\
\hline-5.627 & $2.21 \times 10^{28}$ & $1.4 \times 10^{28}$ \\
\hline-4.627 & $2.17 \times 10^{28}$ & $1.3 \times 10^{28}$ \\
\hline-3.627 & $2.14 \times 10^{28}$ & $1.3 \times 10^{28}$ \\
\hline-2.627 & $2.14 \times 10^{28}$ & $1.2 \times 10^{28}$ \\
\hline-1.627 & $2.07 \times 10^{28}$ & $1.3 \times 10^{28}$ \\
\hline-0.627 & $2.12 \times 10^{28}$ & $1.2 \times 10^{28}$ \\
\hline 0.373 & $2.12 \times 10^{28}$ & $1.3 \times 10^{28}$ \\
\hline 1.373 & $2.18 \times 10^{28}$ & $1.1 \times 10^{28}$ \\
\hline 2.373 & $2.22 \times 10^{28}$ & $7.7 \times 10^{2}$ \\
\hline 3.373 & $2.26 \times 10^{28}$ & $5.0 \times 10^{2}$ \\
\hline 4.373 & $2.15 \times 10^{28}$ & $1.6 \times 10^{28}$ \\
\hline 5.373 & $2.25 \times 10^{28}$ & $1.4 \times 10^{28}$ \\
\hline 6.373 & $1.96 \times 10^{28}$ & $1.5 \times 10^{2}$ \\
\hline 7.373 & $2.09 \times 10^{28}$ & $1.4 \times 10^{28}$ \\
\hline 8.373 & $2.06 \times 10^{28}$ & $2.0 \times 10^{28}$ \\
\hline 9.373 & $2.14 \times 10^{28}$ & $2.0 \times 10^{28}$ \\
\hline 10.373 & $2.34 \times 10^{28}$ & $1.7 \times 10^{28}$ \\
\hline 11.373 & $2.29 \times 10^{28}$ & $1.7 \times 10^{28}$ \\
\hline 12.373 & $2.23 \times 10^{28}$ & $1.7 \times 10^{28}$ \\
\hline 13.373 & $2.33 \times 10^{28}$ & $1.6 \times 10^{28}$ \\
\hline 14.373 & $2.34 \times 10^{28}$ & $1.2 \times 10^{28}$ \\
\hline 15.373 & $2.41 \times 10^{28}$ & $1.1 \times 10^{28}$ \\
\hline 16.373 & $2.14 \times 10^{28}$ & $1.0 \times 10^{28}$ \\
\hline 17.373 & $2.20 \times 10^{28}$ & $1.0 \times 10^{28}$ \\
\hline 18.373 & $2.19 \times 10^{28}$ & $9.8 \times 10^{2}$ \\
\hline 19.373 & $2.32 \times 10^{28}$ & $9.5 \times 10^{2}$ \\
\hline 20.373 & $2.41 \times 10^{28}$ & $9.0 \times 10^{2}$ \\
\hline
\end{tabular}

Table 5

(Continued)

\begin{tabular}{ccc}
\hline \hline$\Delta T$ (days) & $Q\left(\mathrm{~s}^{-1}\right)$ & $\Delta Q\left(\mathrm{~s}^{-1}\right)$ \\
\hline 21.373 & $2.62 \times 10^{28}$ & $7.9 \times 10^{27}$ \\
22.373 & $1.81 \times 10^{28}$ & $1.3 \times 10^{28}$ \\
23.373 & $1.76 \times 10^{28}$ & $1.2 \times 10^{28}$ \\
24.373 & $1.71 \times 10^{28}$ & $1.2 \times 10^{28}$ \\
25.373 & $2.09 \times 10^{28}$ & $9.1 \times 10^{27}$ \\
26.373 & $1.87 \times 10^{28}$ & $9.6 \times 10^{27}$ \\
27.373 & $1.65 \times 10^{28}$ & $9.3 \times 10^{27}$ \\
28.373 & $1.46 \times 10^{28}$ & $8.6 \times 10^{27}$ \\
29.373 & $1.57 \times 10^{28}$ & $1.1 \times 10^{28}$ \\
30.373 & $1.56 \times 10^{28}$ & $9.6 \times 10^{27}$ \\
31.373 & $1.63 \times 10^{28}$ & $8.1 \times 10^{27}$ \\
32.373 & $1.31 \times 10^{28}$ & $8.6 \times 10^{27}$ \\
33.373 & $1.35 \times 10^{28}$ & $8.3 \times 10^{27}$ \\
34.373 & $1.44 \times 10^{28}$ & $7.7 \times 10^{27}$ \\
35.373 & $1.60 \times 10^{28}$ & $6.6 \times 10^{27}$ \\
\hline
\end{tabular}

Notes.

$\Delta T$ : time from perihelion 1997 May 6.6272, in days for each deconvolved value,

$Q$ : water production rates for each image $\left(\mathrm{s}^{-1}\right)$, $\Delta Q: 1 \sigma$ formal uncertainty $\left(\mathrm{s}^{-1}\right)$.

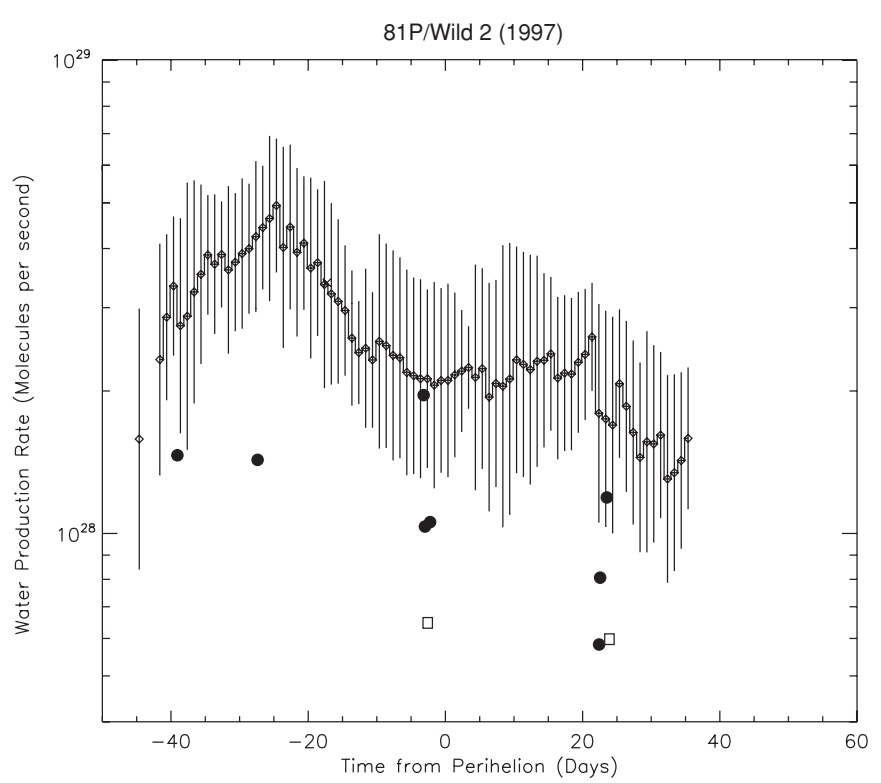

Figure 5. Daily average water production rates of comet $81 \mathrm{P} /$ Wild 2 . The small diamonds give the deconvolved TRM water production rates obtained from the SWAN observations. The vertical lines give the internal uncertainties. The filled circles give water production rates from the $\mathrm{O}\left({ }^{1} \mathrm{D}\right)$ observations by Fink (2009) and the open square give the water production rates from the ground-based $\mathrm{OH}$ photometry by Farnham \& Schleicher (2005).

In Figure 5 note the strong pre- to post-perihelion asymmetry in the water production rate. The production rate is about a factor of two higher at 25 days before perihelion (near the peak) than after perihelion. This same general behavior has been noted before in observations of $\mathrm{OH}$ (a water proxy) but is not seen in the common carbon-bearing radicals $\mathrm{CN}, \mathrm{C}_{2}$, and $\mathrm{C}_{3}$ by Farnham \& Schleicher (2005), whose values were based on ground-based photometry. They noted a peak in the $\mathrm{OH}$ production rate (as well as other components) some 80 days before perihelion. But whereas they have much wider coverage in time from perihelion, they do not actually have measurements from any apparition during this time period we see the peak near 25 days before perihelion. 
Fink (2009) has calculated water production rates from the $6300 \AA \mathrm{O}\left({ }^{1} \mathrm{D}\right)$ emission in $81 \mathrm{P} /$ Wild 2 during the 1997 apparition and his results are generally consistent with, but systematically larger than, those from Farnham \& Schleicher (2005) from the same apparition where they overlap. All observations show the pre- to post-perihelion asymmetry seen also in the SWAN results. It is noteworthy that the SWAN results are systematically a factor of 2-2.5 higher than all the ground-based measurements. The SWAN results shown here are generally consistent with the previous, and totally independent Haser model analysis of Mäkinen et al. (2001) after taking into account the new SWAN calibration factor of 1.55. As mentioned elsewhere a systematic factor of 2-2.5 difference from the ground-based $\mathrm{OH}$ photometry is not unusual and is similar to that for the case of 19P/Borrelly. It is less typical that the water production rates determined from the $\mathrm{O}\left({ }^{1} \mathrm{D}\right)$ measurements from spectrophotometric survey of Fink (2009) are systematically this much lower than the SWAN results.

\section{103P/HARTLEY 2}

Comet 103P/Hartley 2 was the target comet for a flyby during the extended EPOXI mission of the Deep Impact Discovery mission that impacted, observed, and flew by comet 9P/ Tempel 1 (A'Hearn \& Combi 2007) in 2005 July. SWAN observations of 9P/Tempel 1 were already discussed previously by Mäkinen et al. (2007). Hartley 2 is a typical comet in both the A'Hearn et al. (1995) photometry and Fink (2009) spectrophotometry classification surveys, having 1P/Halleylike relative abundances of the carbon radicals, $\mathrm{CN}, \mathrm{C}_{2}$, and $\mathrm{C}_{3}$ as well as $\mathrm{NH}$ and $\mathrm{NH}_{2}$ compared to water. Infrared Space Observatory observations by Crovisier et al. (1999) have determined the $\mathrm{CO}_{2} / \mathrm{H}_{2} \mathrm{O}$ ratio near perihelion in 1997 to be 10\%. The results presented here are all based on the 1997 apparition and information available by the middle of 2010 . The revisions to this paper are being done just after the EPOXI flyby and as press releases and partial preliminary versions of results of the 2010 have been made public. As none of those results have been formally reviewed or published, they will not be part of this paper.

Observations with the Spitzer Space Telescope Infrared Spectrograph by Lisse et al. (2009) made months after the most recent aphelion were analyzed to remove the contributions of the faint trail projected on the sky plane along the comet's orbital direction to reveal a small and dark nucleus having a very small effective radius of $0.57 \pm 0.08 \mathrm{~km}$ and a geometric albedo of only $0.028 \pm 0.009$. With a water production rate of $3 \times$ $10^{28}$ molecules $\mathrm{s}^{-1}$ near perihelion, which as we show below agrees well with our observations, Lisse et al. suggest that nearly $100 \%$ of the surface area is actively emitting volatiles at perihelion. This would put 103P/Hartley 2 in a group of short-period comets with $46 \mathrm{P} /$ Wirtanen and the active fragments of $73 \mathrm{P} /$ Schwassman-Wachmann 3 as short-period comets with a large active fractional surface (A'Hearn et al. 1995).

SOHO SWAN observations of 103P/Hartley 2 were taken from full-sky images during its 1997-1998 apparition. During the 2004 apparition, the comet was too close to the Sun in the sky for even the SWAN instrument to observe. In 1997 the comet reached its perihelion of 1.032 AU on 199722.0173 December. SWAN obtained useful observations from 1997 November 5 until 1998 February 20. This corresponds to fairly continuous coverage from $1.2 \mathrm{AU}$ before perihelion through 1.3 AU afterward. Table 6 gives the observational circumstances for the 1997 apparition and the results of the single-image
Table 6

Comet 103P/Hartley 2: Observational Circumstances and Single-image Water Production Rates

\begin{tabular}{|c|c|c|c|c|c|}
\hline$\Delta T$ (days) & $r(\mathrm{AU})$ & $\Delta(\mathrm{AU})$ & $g\left(\mathrm{~s}^{-1}\right)$ & $Q\left(\mathrm{~s}^{-1}\right)$ & $\Delta Q\left(\mathrm{~s}^{-1}\right)$ \\
\hline-50.518 & 1.246 & 1.015 & 0.001577 & $6.57 \times 10^{27}$ & $1.3 \times 10^{27}$ \\
\hline-47.532 & 1.224 & 1.007 & 0.001579 & $8.97 \times 10^{27}$ & $9.4 \times 10^{26}$ \\
\hline-45.483 & 1.21 & 1.002 & 0.00158 & $1.95 \times 10^{28}$ & $1.3 \times 10^{27}$ \\
\hline-43.559 & 1.196 & 0.996 & 0.001572 & $1.48 \times 10^{28}$ & $7.1 \times 10^{26}$ \\
\hline-42.504 & 1.189 & 0.993 & 0.001572 & $1.36 \times 10^{28}$ & $7.5 \times 10^{26}$ \\
\hline-36.559 & 1.151 & 0.974 & 0.001566 & $1.08 \times 10^{28}$ & $8.1 \times 10^{26}$ \\
\hline-33.549 & 1.134 & 0.963 & 0.001559 & $1.08 \times 10^{28}$ & $1.7 \times 10^{27}$ \\
\hline-31.337 & 1.121 & 0.955 & 0.001561 & $1.91 \times 10^{28}$ & $1.5 \times 10^{27}$ \\
\hline-29.413 & 1.111 & 0.948 & 0.001554 & $2.69 \times 10^{28}$ & $2.2 \times 10^{27}$ \\
\hline-28.358 & 1.106 & 0.944 & 0.001555 & $2.81 \times 10^{28}$ & $4.2 \times 10^{26}$ \\
\hline-26.358 & 1.096 & 0.937 & 0.001556 & $2.92 \times 10^{28}$ & $2.9 \times 10^{26}$ \\
\hline-24.327 & 1.087 & 0.929 & 0.001549 & $2.29 \times 10^{28}$ & $8.8 \times 10^{26}$ \\
\hline-19.574 & 1.068 & 0.911 & 0.001546 & $1.88 \times 10^{28}$ & $2.2 \times 10^{27}$ \\
\hline-17.525 & 1.061 & 0.904 & 0.00154 & $2.12 \times 10^{28}$ & $2.7 \times 10^{26}$ \\
\hline-15.803 & 1.056 & 0.897 & 0.001542 & $2.60 \times 10^{28}$ & $2.1 \times 10^{26}$ \\
\hline-12.487 & 1.047 & 0.885 & 0.001537 & $2.64 \times 10^{28}$ & $2.3 \times 10^{26}$ \\
\hline-10.432 & 1.0 & 0.8 & 0.00 & $3.25 \times 10^{28}$ & $2.1 \times 10^{26}$ \\
\hline-8.515 & 1.039 & 0.87 & 0.00 & $2.46 \times 10^{28}$ & $2.6 \times 10^{26}$ \\
\hline-6.523 & 1.036 & 0.864 & 0.001531 & $4.31 \times 10^{28}$ & $2.9 \times 10^{26}$ \\
\hline-1.281 & 1.032 & 0.849 & 0.001528 & $3.23 \times 10^{28}$ & $1.8 \times 10^{26}$ \\
\hline-0.226 & 1.032 & 0.846 & 0.001529 & $3.34 \times 10^{28}$ & $2.1 \times 10^{26}$ \\
\hline 1.76 & 1.032 & 0.841 & 0.00153 & $3.54 \times 10^{28}$ & $1.9 \times 10^{26}$ \\
\hline 3.842 & 1.033 & 0.836 & 0.001529 & $3.48 \times 10^{28}$ & $8.4 \times 10^{25}$ \\
\hline 5.75 & 1.035 & 0.832 & 0.00153 & $3.68 \times 10^{28}$ & $1.5 \times 10^{26}$ \\
\hline 8.778 & 1.039 & 0.827 & 0.00153 & $2.65 \times 10^{28}$ & $2.1 \times 10^{26}$ \\
\hline 10.833 & 1.043 & 0.824 & 0.001531 & $2.45 \times 10^{28}$ & $2.3 \times 10^{26}$ \\
\hline 12.75 & 1.047 & 0.822 & 0.001531 & $2.91 \times 10^{28}$ & $1.9 \times 10^{26}$ \\
\hline 13.805 & 1.05 & 0.821 & 0.001532 & $2.53 \times 10^{28}$ & $1.9 \times 10^{26}$ \\
\hline 15.806 & 1.056 & 0.821 & 0.001534 & $2.36 \times 10^{28}$ & $1.8 \times 10^{26}$ \\
\hline 17.861 & 1.062 & 0.8 & 0.001 & $2.23 \times 10^{28}$ & $1.8 \times 10^{26}$ \\
\hline 19.778 & 1.069 & 0.821 & 0.001537 & $2.64 \times 10^{28}$ & $2.0 \times 10^{26}$ \\
\hline 22.82 & 1.081 & 0.824 & 0.001541 & $2.26 \times 10^{28}$ & $1.8 \times 10^{26}$ \\
\hline 24.875 & 1.09 & 0.827 & 0.001542 & $2.02 \times 10^{28}$ & $1.9 \times 10^{26}$ \\
\hline 26.597 & 1.097 & 0.83 & 0.001546 & $2.45 \times 10^{28}$ & $1.7 \times 10^{26}$ \\
\hline 29.975 & 1.114 & 0.838 & 0.001552 & $2.37 \times 10^{28}$ & $1.6 \times 10^{26}$ \\
\hline 33.807 & 1.135 & 0.851 & 0.001554 & $2.00 \times 10^{28}$ & $1.9 \times 10^{26}$ \\
\hline 36.334 & 1.15 & 0.861 & 0.001562 & $1.52 \times 10^{28}$ & $2.0 \times 10^{26}$ \\
\hline 38.39 & 1.163 & 0.87 & 0.001563 & $1.58 \times 10^{28}$ & $2.2 \times 10^{26}$ \\
\hline 40.32 & 1.175 & 0.88 & 0.001571 & $1.56 \times 10^{28}$ & $2.1 \times 10^{26}$ \\
\hline 43.375 & 1.195 & 0.897 & 0.001573 & $1.36 \times 10^{28}$ & $2.7 \times 10^{26}$ \\
\hline 50.377 & 1.245 & 0.944 & 0.001585 & $2.20 \times 10^{28}$ & $2.7 \times 10^{26}$ \\
\hline 52.433 & 1.26 & 0.96 & 0.001595 & $2.44 \times 10^{28}$ & $3.0 \times 10^{27}$ \\
\hline 55.396 & 1.283 & 0.985 & 0.001597 & $1.11 \times 10^{28}$ & $7.4 \times 10^{26}$ \\
\hline 57.405 & 1.298 & 1.002 & 0.001598 & $1.49 \times 10^{28}$ & $2.3 \times 10^{27}$ \\
\hline 59.461 & 1.315 & 1.021 & 0.0016 & $1.18 \times 10^{28}$ & $1.1 \times 10^{27}$ \\
\hline 61.377 & 1.33 & 1.04 & 0.001611 & $1.24 \times 10^{28}$ & $4.2 \times 10^{26}$ \\
\hline
\end{tabular}

Notes.

$\Delta T$ : time from perihelion 1997 December 22.0173, in days for each SWAN image,

$r$ : heliocentric distance (AU),

$\Delta$ : geocentric distance (AU),

$g$ : solar Ly $\alpha$ g-factor (photons s ${ }^{-1}$ ),

$Q$ : water production rates for each image $\left(\mathrm{s}^{-1}\right)$, $\Delta Q: 1 \sigma$ formal uncertainty $\left(\mathrm{s}^{-1}\right)$.

analysis of water production rates. The water production rates are plotted as a function of heliocentric distance both before and after perihelion in Figure 6. In each case, the variation of production rates with heliocentric distance is quite steep, putting it into the small group of comets with very steep slopes along with 19P/Borrelly. The best-fit power-law water production variations for pre- and post-perihelion, respectively, are $3.9 \times$ $10^{28} r^{-6.6 \pm 0.1}$ and $3.1 \times 10^{28} r^{-3.2 \pm 0.1}$, in molecules per second. 


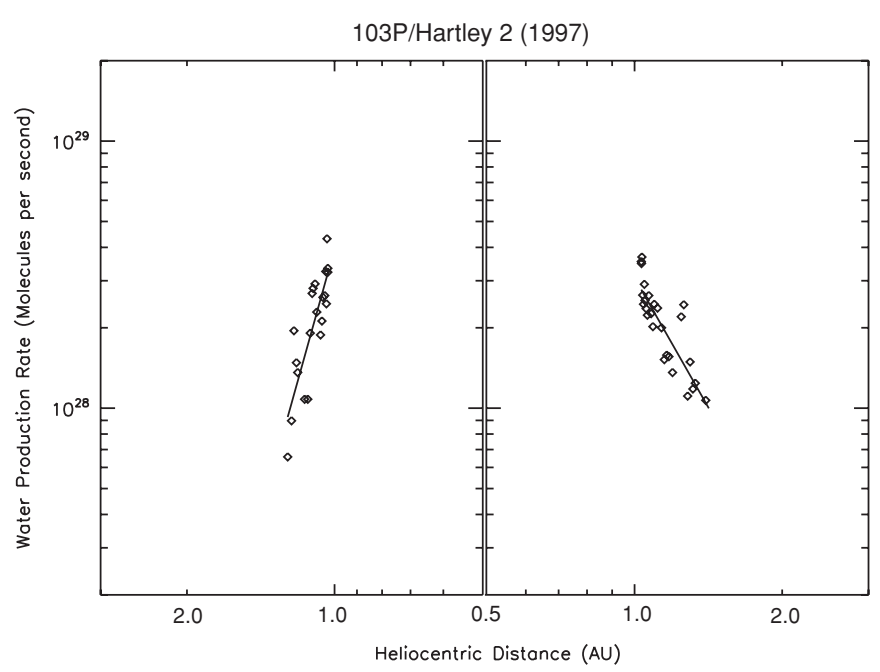

Figure 6. Single-image water production rates and fitted power-law distributions for comet $103 \mathrm{P} /$ Hartley 2 . The straight lines give the best-fit power-law variations of water production with heliocentric distance, $r$, of the form $3.9 \times$ $10^{28} r^{-6.6 \pm 0.1}$ and $3.1 \times 10^{28} r^{-3.2 \pm 0.1}$.

Figure 7 shows the water production rate plotted as a function of time from perihelion in days. The variation is fairly steady. The production rate rises more rapidly before perihelion than it falls afterward, and reached its highest value 6 days before perihelion. There are factor of $\sim 2$ "outbursts" of activity relative to the overall trend in variation at $T=-30$ days, -6 days and +50 days that appear to last several days each. These outbursts are considerably longer than the rotation period $(\sim 18 \mathrm{hr})$. The large SWAN FOV and the fairly long photodissociation times for producing $\mathrm{H}$ from $\mathrm{H}_{2} \mathrm{O}$ and $\mathrm{OH}$ mean that $\mathrm{SWAN}$ measurements would not be sensitive to rotational time variations.

A steep slope in the heliocentric distance variation of the water production rate of a comet is often indicative of a seasonal effect. This could be based on an irregular shape of the nucleus and its changing orientation of the spin axis and its shape with respect to solar illumination as the comet moves in its orbit. It can also indicate that there is a non-uniform distribution of active areas on the surface of the nucleus with respect to the spin axis and its orientation with respect to solar illumination as the comet moves in its orbit. Deviations of water production rate from an ideal $1 / r^{2}$ variation, when comets are less that about $2.5 \mathrm{AU}$ from the Sun are usually interpreted as indicating a seasonal variation of nucleus cross section or distribution of active areas on the surface is occurring.

Whatever the shape and whatever the orientation of the spin axis of the nucleus of Hartley 2 are, the cross-sectional area of the nucleus in view during the 5.5 AU Spitzer observations should be approximately the same as the one illuminated by the Sun when the comet is near perihelion. Actually it should be nearly exactly the opposite view. Therefore, the conclusion of Lisse et al. that the small nucleus must be highly active $(50 \%-100 \%)$ near perihelion is quite reasonable given the small cross-sectional area of the nucleus and its large water production rate near perihelion $\left(\sim 3 \times 10^{28} \mathrm{~s}^{-1}\right)$.

However, the steep production rate variation found here $\left(r^{-6.6}\right.$ before perihelion and $r^{-3.2}$ afterward) implies that either the cross section or the distribution of active areas is quite different between perihelion and roughly +50 days and -50 days away from perihelion, corresponding to the first and last SWAN observations, or that large physical changes in active areas happen during one apparition. During this time, the comet

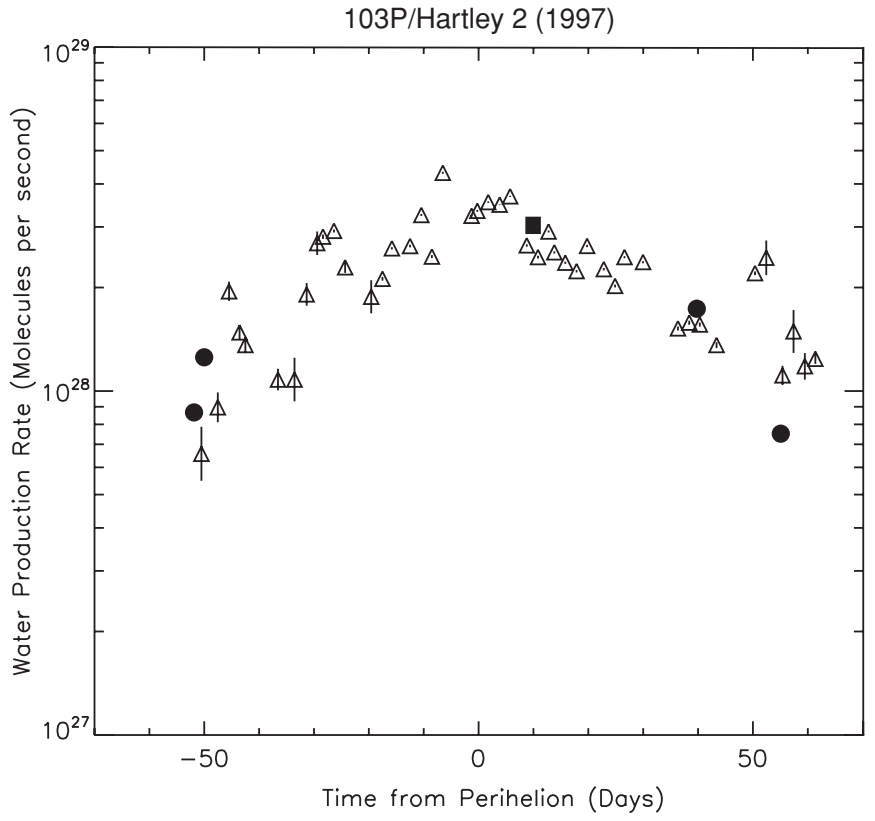

Figure 7. Single-image water production rates of comet 103P/Hartley 2 as a function of time. The water production rates from the SWAN measurements are shown as the triangles with very small internal uncertainties as the short lines. The filled circles are water production rates from the $\mathrm{O}\left({ }^{1} \mathrm{D}\right)$ observations of Fink (2009) made also during the 1997 apparition. The one filled square 10 days after perihelion is from the ground-based $\mathrm{OH}$ measurements in the photometric survey of A'Hearn et al. (1995). We find good agreement among these and the SWAN results. Of note are the elevated production rates near $T=$ $-30,-6$ and +50 days from perihelion.

moves over about $90^{\circ}$ of true anomaly or from $-45^{\circ}$ and $+45^{\circ}$ relative to perihelion. If the former, it would imply that the cross sections illuminated by the Sun 50 days before and after perihelion are a factor of about 2.2 smaller than that facing the Sun at perihelion and approximately in view during the 5.5 AU Spitzer observation. This could then require that the comet is fairly ellipsoidal and the mean radius of the comet facing the Sun 50 days before and after perihelion is about 1.5 times smaller than that determined by the Spitzer observation, or having an effective radius of only $0.38 \mathrm{~km}$. However, all that the production rate variation constrains is the rotationally averaged effective active area of the comet as a function of orbital position. Alternatively, $0.38 \mathrm{~km}$ could be just the radius of the active area illuminated by the Sun at +50 and -50 days from perihelion, and not necessarily place any constraint on physical cross section of the nucleus illuminated 50 days before or after perihelion.

A possible explanation is that Hartley 2, like Borrelly, might have a spin axis that is nearly sunward near perihelion and that the main active region is near this pole and illuminated near perihelion enhancing its activity beyond the normal rotationally averaged sublimation rate. Furthermore, this aspect would then have an effective physical radius as well as an active area effective radius of $0.58 \mathrm{~km}$. This would be consistent with this region receiving much less illumination when the comet's axis is at an inclination of $45^{\circ}$ at +50 and -50 days from perihelion. This would be the Borrelly-like model, which has a broad flat surface on the face of the nucleus that contains the spin axis and that faces the Sun around perihelion. A flat sunward face near perihelion both can enhance illumination angle as well as its drop off with decreasing illumination as the comet progresses in its orbit. 
Table 7

Volatile Composition for Three Water Variation Groups

\begin{tabular}{|c|c|c|c|c|}
\hline Comet & $Q_{1}\left(\mathrm{H}_{2} \mathrm{O}\right)^{\mathrm{a}}$ & $p^{\mathrm{b}}$ & $\mathrm{CO} / \mathrm{H}_{2} \mathrm{O}$ & $\mathrm{C}_{2} \mathrm{H}_{6} / \mathrm{H}_{2} \mathrm{O}$ \\
\hline \multicolumn{5}{|c|}{ Steep slope } \\
\hline 21P/Giacobini-Zinner (post- $q$ ) & $6.8 \times 10^{28}$ & $-11.9^{c}$ & $0.5 \%-10 \%$ & $0.2 \%-0.6 \%$ \\
\hline 19P/Borrelly & $3.3 \times 10^{29 \mathrm{~d}}$ & -6.1 & $<15 \%$ & - \\
\hline C 2001 A2 LINEAR & $1.1 \times 10^{29}$ & -4.5 & $1.5 \%$ & $1.6 \%$ \\
\hline 103P/Hartley 2 & $3.1 \times 10^{28}$ & -3.6 & $<1 \%{ }^{\mathrm{e}}$ & - \\
\hline C 1999 T1 McNaught-Hartley & $7.0 \times 10^{29}$ & -3.3 & $15 \%$ & $0.5 \%$ \\
\hline \multicolumn{5}{|c|}{ Moderate slope } \\
\hline C 1995 OI Hale-Bopp & $2.0 \times 10^{31}$ & -2.6 & $23 \%$ & $0.6 \%$ \\
\hline C 1999 H1 Lee & $2.5 \times 10^{29}$ & -2.7 & $2 \%-4 \%$ & $0.7 \%$ \\
\hline P153/Ikeya-Zhang & $2.4 \times 10^{29}$ & -2.7 & $4 \%$ & $0.6 \%$ \\
\hline C 1996 B2 Hyakutake & $4.2 \times 10^{29}$ & -2.1 & $19 \%-30 \%$ & $0.6 \%$ \\
\hline C 2002 T7 LINEAR & $6.0 \times 10^{29}$ & -2.0 & $2 \%-3 \%$ & $\sim 0.5 \%$ \\
\hline \multicolumn{5}{|c|}{ Shallow slope } \\
\hline C 2001 Q4 NEAT & $4.6 \times 10^{29}$ & -1.7 & $3 \%-9 \%$ & $\sim 0.5 \%$ \\
\hline C 2006 P1 McNaught & $1.2 \times 10^{30}$ & -1.7 & $1.5 \%-1.8 \%{ }^{\mathrm{f}}$ & $0.36-0.6^{\mathrm{f}}$ \\
\hline 21P/Giacobini-Zinner (pre- $q$ ) & $5.9 \times 10^{28}$ & $-1.7^{\mathrm{c}}$ & $0.5 \%-10 \%$ & $0.2 \%-0.6 \%$ \\
\hline C 1999 S4 LINEAR & $2.9 \times 10^{28}$ & -1.6 & $0.6 \%$ & $0.1 \%$ \\
\hline C 2001 WM1 LINEAR & $1.4 \times 10^{29}$ & -1.4 & $1 \%$ & $0.5 \%$ \\
\hline
\end{tabular}

Notes.

${ }^{\text {a }}$ Average pre-post water production rate at $1 \mathrm{AU}$.

b Average pre-post power-law exponent.

c The power law for 21P/Giacobini-Zinner was very different for the pre-perihelion and post-perihelion.

d The values of $Q 1$ for $19 \mathrm{P} /$ Borrelly and $21 \mathrm{P} /$ Giacobini-Zinner need to be considered in the light that the perihelion distances of both are greater than $1 \mathrm{AU}$.

e The upper limit for Hartley 2 is from Weaver et al. (1994).

${ }^{\mathrm{f}}$ Composition results for McNaught are from Dello Russo et al. (2009).

Another scenario is that the nucleus is highly ellipsoidal with the largest cross section of the nucleus exposed to the Sun at perihelion (and seen by Spitzer at $5.5 \mathrm{AU}$ ). In this case, the illuminated ellipsoid would have a cross Section 1.5 times smaller (an effective radius of about $0.38 \mathrm{~km}$ ) at a true anomaly of + and $-45^{\circ}$ from perihelion. Rather than the cross section of the ellipsoid of the comet getting smaller it could also be that the parts of the nucleus illuminated at a true anomaly $45^{\circ}$ away from perihelion are lacking in active areas, that is a larger geometrical area but a smaller active area. Unfortunately, some combination of these effects is also possible. In any case, the $r^{-3.6}$ variation means that the effective radius of $0.58 \mathrm{~km}$ either represents its largest cross section or parts of the surface are not nearly as active as $100 \%$ from those parts of the nucleus that are illuminated when the comet is near perihelion.

Meech et al. (2009) have recently reported the results of $H S T$ and various ground-based observations of 103P/Hartley 2 that show a light curve that has uneven maxima and sharp minima. They suggest the nucleus may have an irregular shape that deviates from a simple triaxial ellipsoid. They also find a rotation period for the nucleus of about $16.6 \mathrm{hr}$. The steep drop in water production for the four months before and after perihelion is interesting considering that the activity seems to be fairly large at large heliocentric distances as judged by the presence of a coma and tail at a fairly large heliocentric distance of $4.73 \mathrm{AU}$ by Licandro et al. (2000) in 1993, by Lowry \& Fitzsimmons (2001) at 3.63 AU outbound, and 4.57 AU outbound by Lowry et al (2003) as reported by Mazzotta Epifani et al. (2008). Taken together this indicates that Hartley 2 may have a very complicated shape as well as a complicated pattern of activity all around its orbit.

\section{DISCUSSION}

Water production rates derived from observations of water directly in the infrared as well as from observations of various water photodissociation products including $\mathrm{H} \mathrm{Ly} \alpha$, space-based $\mathrm{OH}$, ground-based $\mathrm{OH}$, radio $\mathrm{OH}$, and $\mathrm{O}\left({ }^{1} \mathrm{D}\right)$ usually exhibit differences from one another. Some of these differences are directly attributable to differences in coma models and/or model parameters (Combi et al. 1993, 2005; Schleicher et al. 1998) that are necessarily used in the analyses. Others are probably attributable to combinations of errors or uncertainties in excitation mechanisms ( $g$-factors), excitation mechanism parameters such as rotational temperature models and excitation quenching, or atmospheric absorption corrections, background subtraction, and solar flux calibration.

The SWAN observations for comet 81P/Wild 2 included in this paper were published earlier using a different model analysis procedure. The new analysis procedure accounts explicitly for the more detailed velocity distribution of the dissociation products as they contribute to the coma, so there are detailed differences between these results and those previously published, but there are no large systematic differences. We have also performed the extraction daily average values of the water production rate from the series of images for this work.

In addition to providing fairly continuous coverage of water production rates in comets for context use by the community, we also have been calculating the heliocentric distance dependence of the water production rate in the form of the standard power-law heliocentric distance in the form $Q=Q(1 \mathrm{AU}) r^{p}$, where $Q(1 \mathrm{AU})$ is the water production rate of the comet at a heliocentric distance of $1 \mathrm{AU}, r$ is the heliocentric distance 
Table 8

Calibration Factors for Past SWAN Observations of Comets

\begin{tabular}{lcc}
\hline \hline \multicolumn{1}{c}{ Comet } & Calibration Factor & Reference \\
\hline C 1996 B2 Hyakutake & 1.55 & Bertaux et al. (1998) and Combi et al. (2005) \\
C 1995 OI Hale-Bopp & 1.55 & Combi et al. (2000) \\
46P/Wirtanen & 1.55 & Bertaux et al. (1999) \\
C 1999 S4 LINEAR & 1.68 & Mäkinen et al. (2001) \\
C 2001 A2 LINEAR & 1.31 & Combi et al. (2008) \\
C 1999 T1 McNaught-Hartley & 2.40 & Combi et al. (2008) \\
C 1999 H1 Lee & 1.68 & Combi et al. (2008) \\
P153/Ikeya-Zhang & 1.31 & Combi et al. (2008) \\
C 2001 WM1 LINEAR & 1.31 & Combi et al. (2008) \\
C 2002 T7 LINEAR & 1.31 & Combi et al. (2009a) \\
C 2001 Q4 NEAT & 1.31 & Combi et al. (2009a) \\
9P/Tempel 1 & 1.31 & Mäkinen et al. (2007) \\
C 2006 P1 McNaught & 1.31 & Neugebauer et al. (2007) \\
17P/Holmes & 1.31 & Combi et al. (2007) \\
C 2007 N1 Lulin & 1.31 & Combi et al. (2009b) \\
\hline
\end{tabular}

in $\mathrm{AU}$, and $p$ is the power-law exponent. In previous work this has been done exclusively for long-period comets. With this study we are able to add two Jupiter family comets to our compilation. The results for $81 \mathrm{P} /$ Wild 2 did not lend themselves to yield a useful fit to a power law because of the peculiar and likely seasonal variation within the couple of months around perihelion where we could obtain useful observations.

Table 7 shows the latest compilation of SWAN observations of comets for the three power-law exponent groups: steep, nominal and shallow (Combi et al. 2008, 2009a). These are compared with the production rate reduced to a heliocentric distance of $1 \mathrm{AU}$ and to two measures of the volatile composition of the comets as measured by the $\mathrm{CO} / \mathrm{H}_{2} \mathrm{O}$ and $\mathrm{C}_{2} \mathrm{H}_{6} / \mathrm{H}_{2} \mathrm{O}$ ratios. $\mathrm{CO}$ and $\mathrm{C}_{2} \mathrm{H}_{6}$ were chosen by Combi et al. (2008) as a representative subset of cometary volatiles, with $\mathrm{CO}$ being highly variable $(<1 \%-30 \%$ compared with water) from comet to comet (Bockelée-Morvan et al. 2004) and $\mathrm{C}_{2} \mathrm{H}_{6}$ being reasonably steady ( $\sim 0.6 \%$ compared to water) in most comets. These are included here primarily for consistency with previous papers. So far there has not been any definite correlation among production rate level, power-law slope, $\mathrm{CO}$ or $\mathrm{C}_{2} \mathrm{H}_{6}$ abundance, or any other major species for that matter. The results for the previously presented comet are explained in these two papers, as are the sources for the composition observations. Those for the comets presented in this paper have been mentioned already in the previous sections of this paper about each comet. $21 \mathrm{P} /$ Giacobini-Zinner is very different between pre- and postperihelion with a shallow slope and generally higher production rates before perihelion and a precipitous drop in production rate after perihelion.

For the new results added in this work it is noteworthy that 19P/Borrelly has the steepest variation slope of any comet both pre- and post-perihelion, in agreement with previous observations, that 103P/Hartley 2 has the next steepest consistent variation. The steepness of the variation of the production rate of $103 \mathrm{P} /$ Hartley 2 , in combination with its production rate of $3 \times$ $10^{28} \mathrm{~s}^{-1}$ at perihelion, and measured geometrical cross section with an effective radius of $0.58 \mathrm{~km}$ near aphelion, places some interesting constraints on the possible shape of the comet, the orientation of the spin axis, and the distribution of active areas around its surface.

Finally, Table 8 gives multiplicative correction factors for previously published values of comet water production rates determined from SWAN observations beginning with the 1996 observations of 1996 B2 Hyakutake that started only a few weeks after $\mathrm{SOHO}$ was launched.

SOHO is an international cooperative mission between ESA and NASA. M. Combi acknowledges support from grant NNX06AB60G from the Outer Planets Research Program and NNX09AN34G of the Planetary Mission Data Analysis Program. J.-L. Bertaux and E. Quémerais acknowledge support from CNRS and CNES. J. T. T. Mäkinen was supported by the Finnish Meteorological Institute. We obtained cometary ephemerides and orbital elements from the JPL Horizons Web site. The composite solar Ly $\alpha$ data were taken from the LASP Web site http://lasp.colorado.edu/lisird/lya/lymanalpha.html. We acknowledge the personnel that have been keeping $\mathrm{SOHO}$ and SWAN operational for over 14 years. We also thank Nathaniel Henry who helped with some of the data reduction.

\section{REFERENCES}

A'Hearn, M. F., \& Combi, M. R. 2007, Icarus, 187, 1

A'Hearn, M. F., Millis, R. L., Schleicher, D. G., Osip, D. J., \& Birch, P. V. 1995, Icarus, 118, 223

Beaver, J. E., Wagner, R. M., Schleicher, D. G., \& Lutz, B. L. 1990, ApJ, 360, 696

Bertaux, J. L., Costa, J., Mäkinen, T., Quémerais, E., Lallement, R., Kyrölä, E., \& Schmidt, W. 1999, Planet. Space Sci., 47, 725

Bertaux, J. L., et al. 1995, Sol. Phys., 162, 403

Bertaux, J. L., et al. 1998, Planet. Space Sci., 46, 555

Bockelée-Morvan, D., Biver, N., Colom, P., Crovisier, J., Festou, M. C., Henry, F., Lecacheux, A., \& Weaver, H. A. 2002, Earth Moon Planets, 90, 459

Bockelée-Morvan, D., et al. 2004, Icarus, 167, 113

Brownlee, D. E., et al. 2004, Science, 304, 1764

Clarke, J. T., Lallement, R., Bertaux, J.-L., Fahr, H., Quémerais, E., \& Scherer, H. 1998, ApJ, 499, 482

Combi, M. R., Bertaux, J.-L., Quémerais, E., \& Mäkinen, J. T. T. 2009b, IAU Circ. 9020

Combi, M. R., Bos, B., \& Smyth, W. H. 1993, ApJ, 408, 668

Combi, M. R., \& Feldman, P. D. 1992, Icarus, 97, 260

Combi, M. R., Mäkinen, J. T. T., Bertaux, J.-L., \& Quémerais, E. 2005, Icarus, 177,228

Combi, M. R., Mäkinen, J. T. T., Bertaux, J.-L., Quémerais, E., \& Ferron, S. 2007, IAU Circ. 8905

Combi, M. R., Mäkinen, J. T. T., Henry, N. J., Bertaux, J.-L., \& Quémerais, E. 2008, AJ, 135, 1533

Combi, M. R., Mäkinen, J. T. T., Lee, Y., Bertaux, J.-L., \& Quémerais, E. 2009a, AJ, 137, 4734

Combi, M. R., Reinard, A. A., Bertaux, J.-L., Quémerais, E., \& Mäkinen, T. 2000, Icarus, 144, 191

Combi, M. R., \& Smyth, W. H. 1988, ApJ, 327, 1044 
Combi, M. R., Stewart, A. I. F., \& Smyth, W. H. 1986, Geophys. Res. Lett., 13 385

Crovisier, J., et al. 1999, in The Universe as Seen by ISO, ed. P. Cox \& M. F Kessler (ESA-SP 427; Noordwijk: ESA), 161

Dello Russo, N., Vervack, R. J., Weaver, H. A., Kawakita, H., Kobayashi, H., Biver, N., Bockelée-Morvan, D., \& Crovisier, J. 2009, ApJ, 703, 187

Farnham, T., \& Schleicher, D. G. 2005, Icarus, 173, 153

Fink, U. 2009, Icarus, 301, 311

Fink, U., \& Hicks, M. 1996, ApJ, 459, 729

Konno, I., \& Wyckoff, S. 1989, Adv. Space Res., 9, 163

Licandro, J., Tancredi, G., Lindgren, M., Rickman, H., \& Gil Hutton, R. 2000, Icarus, 147, 161

Lisse, C. M., et al. 2009, PASP, 121, 968

Lowry, S. C., \& Fitzsimmons, A. 2001, A\&A, 365, 204

Lowry, S. C., Fitzsimmons, A., \& Collander-Brown, S. 2003, A\&A, 397, 329

Mäkinen, J. T. T., \& Combi, M. R. 2005, Icarus, 177, 217

Mäkinen, J. T. T., Combi, M. R., Bertaux, J.-L., Quémerais, E., \& Schmidt, W. 2007, Icarus, 187, 109

Mäkinen, J. T. T., Silén, J., Schmidt, W., Kyrölä, E., Summanen, T., Bertaux, J.-L., Quémerais, E., \& Lallement, R. 2001, Icarus, 152, 268

Mazzotta Epifani, E., Palumbo, P., Capria, M. T., Cremonese, G., Fulle, M., \& Colangeli, L. 2008, MNRAS, 390, 265

McClintock, W. E., Rottman, G. J., \& Woods, T. N. 2005, Sol. Phys., 230, 225
McFadden, L., et al. 1987, Icarus, 69, 329

Meech, K., et al. 2009, AAS DPS Meeting, 41, 20.07

Mumma, M., DiSanti, M. A., Dello Russo, N., Magee-Sauer, K., \& Rettig, T. W. 2000, ApJ, 53, L155

Neugebauer, M., et al. 2007, ApJ, 667, 1262

Quémerais, E., \& Bertaux, J.-L. 2002, in Radiometric Calibration of the SWAN Instrument, in The Radiometric Calibration of SOHO, ed. A. Pauluhn, M. C. E. Huber, \& R. von Steiger (ESA SR-002; Noordwijk: ESA), 203

Quémerais, E., Lallement, R., Sandel, B. R., \& Clarke, J. 2009, Space Sci. Rev., 143,151

Schleicher, D. G., Millis, R. L., \& Birch, P. V. 1987, A\&A, 187, 531

Schleicher, D. G., Millis, R. L., \& Birch, P. V. 1998, Icarus, 132, 397

Schleicher, D. G., Woodney, L. M., \& Millis, R. L. 2003, Icarus, 162, 415

Sekanina, Z. 1985, AJ, 90, 827

Sekanina, Z., \& Yeomans, D. K. 1985, AJ, 90, 2335

Soderblom, L. A., et al. 2002, Science, 296, 1087

von Rosenvinge, T. T., Brandt, J. C., \& Farquhar, R. W. 1986, Science, 232, 353

Weaver, H. A., Feldman, P. D., McPhate, J. B., A'Hearn, M. F., Arpigny, C., \& Smith, T. E. 1994, ApJ, 442, 374

Weaver, H. A., Stern, S. A., \& Parker, J. Wm 2003, AJ, 126, 444

Weaver, H. A., et al. 1999, Icarus, 142, 482

Woods, T. N., Tobiska, W. K., Rottman, G. J., \& Worden, J. R. 2000, J. Geophys. Res., 105, 27195

Woods, T. N., et al. 1996, J. Geophys. Res., 101, 9541 